
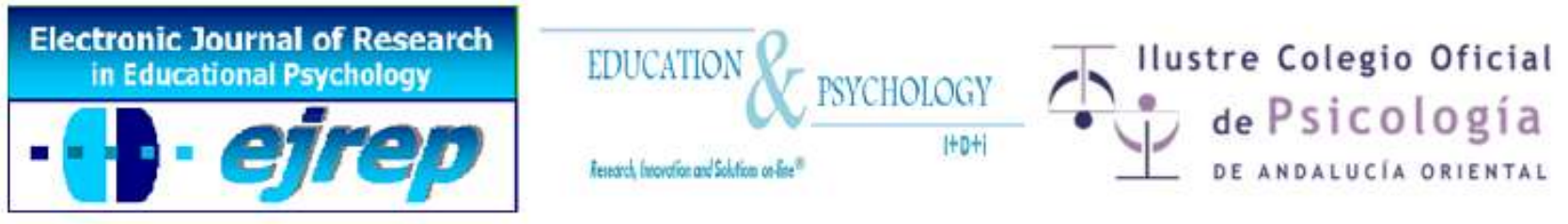

\title{
La resolución de problemas matemáticos a través del análisis secuencial de procesos
}

\author{
Codina, A. ${ }^{1,2}$, Cañadas, M. C. ${ }^{2}$, Castro, E. ${ }^{2}$ \\ ${ }^{1}$ Dpto. de Educación, Universidad de Almería, Almería \\ ${ }^{2}$ Dpto. de Didáctica de la Matemática, Universidad de Granada, Granada
}

\section{España}

Correspondencia: Antonio Codina Sánchez, Dpto. de Educación, Edificio A, Universidad de Almería, C.P. 04120, Almería, España. E-mail: acodina@ual.es

(C) Education \& Psychology I+D+i and Ilustre Colegio Oficial de la Psicología de Andalucía Oriental (Spain) 


\section{Resumen}

Introducción. La perspectiva macroscópica es uno de los marcos desde los que se aborda la investigación sobre resolución de problemas en la enseñanza de las matemáticas. Nuestro estudio, dentro de esta perspectiva, se enmarca dentro de los estadios del pensamiento en la resolución de problemas matemáticos, ofreciendo un enfoque innovador porque aplicamos el análisis secuencial de procesos y la técnica de coordenadas polares para estudiar las relaciones secuenciales e interrelaciones globales entre los distintos estadios en la resolución de problemas matemáticos.

Método. Esta investigación se basa en la metodología observacional, adoptando como unidad de análisis el conjunto de procesos observables de una pareja de estudiantes resolviendo un problema matemático. La calidad de la información (fiabilidad intra e interobservador y el test de independencia de la Chí-cuadrado) está garantizada permitiendo aplicar el análisis secuencial así como la técnica de coordenadas polares.

Resultados. Presentamos dos niveles de concreción, uno para cada sujeto y otro para la pareja. Analizamos el conjunto de estadísticas básicas; periodos de trabajo colaborativo y paralelo; probabilidades de transición, secuencias o cadenas significativas, traslaciones de ejecución y el conjunto de mapas de relaciones globales entre los diferentes estadios. Los resultados permiten describir y analizar el comportamiento de los sujetos y la pareja durante el proceso de resolución así como el trabajo colaborativo puesto en juego.

Discusión. El estudio refleja una nueva aproximación para investigar las interrelaciones entre las etapas de resolución de problemas y el trabajo colaborativo macroscópicamente, abriendo un camino de investigación en educación matemática. Los dos niveles de concreción permiten obtener resultados que describen las influencias individuales en el proceso de resolución conjunto, concretando con mayor profundidad en las interrelaciones surgidas entre los sujetos y el trabajo colaborativo puesto en juego. El estudio muestra el potencial de este análisis para el estudio de las dificultades en el aprendizaje de la resolución de problemas matemáticos.

Palabras Clave. Estadios en resolución de problemas matemáticos, traslación de ejecución, análisis secuencial, coordenadas polares.

Recibido: 04/06/14 Aceptación Inicial: 15/08/14 Aceptación final: 10/03/15 


\title{
Mathematical problem solving through sequential process analysis
}

\begin{abstract}
Introduction. The macroscopic perspective is one of the approaches to tackled research on problem solving. Our study, from this perspective, is focused in the thinking stages when solving mathematical problems, offering an innovative standpoint because we apply the sequential analysis process and the polar coordinate technique to study the sequential relationships and the global interrelationships between the different stages of mathematical problem solving.

Method. This study is based on observational methodology, using as unit of analysis the set of processes that we can observe from a pair of students when solving a mathematical problem. The quality of the information (reability intra and inter-observer and the Chi-squared independent test) is guaranteed, so it is allowed to apply the sequential analisys and also the polar coordinates technique.
\end{abstract}

Results. We present two precision levels, one per each student, and the other for each pair. We then obtain the set of basic statistics; the periods of collaborative work and parallel work; the transition probabilities, the signifivative sequences or chains, the traslations of realisations and the set of maps that includes the global relationships between different stages. These results allow to describe and analyse the behabiour of students and pair when solving mathematical problems and the collaborative work performed.

Discussion. This paper reflects a new approach to research the interrelationships that arise between the stages of problem solving and the collaborative work from a macroscopic viewpoint. This fact constitutes a new methodology in mathematics education. The two precision levels allow to obtain results that describe the individual influence in the whole process of problem solving, describing more deeply the interrelationships emerged between students and the collaborative work performed. Also this paper shows the potential of this kind of analysis for studying the learning difficulties in solving mathematical problems.

Keywords. stages in solving mathematical problems, execution traslations, sequential analysis, polar coordinates 


\section{Introducción}

La resolución de problemas matemáticos es un tema de investigación tradicional en Educación Matemática especialmente desarrollada durante las tres últimas décadas. En este sentido, se ha generado un extenso y heterogéneo cuerpo de conocimiento con orientaciones metodológicas y análisis diversos (Castro, 2008; Kilpatrick, 1992; Törner, Reiss, y Schoenfeld, 2007). Actualmente, las posibilidades ofrecidas por las tecnologías digitales están propiciando la apertura de nuevas líneas de investigación que relacionan la interacción humanomáquina, el diseño de sistemas interactivos o el diseño de learning-objects con la resolución de problemas matemáticos (Codina, Cañadas y Castro, 2010; Dix, Finlay, Abowd, y Beale, 2004; Mgombelo y Buteau, 2010).

Si bien es cierto que las investigaciones en resolución de problemas matemáticos tienen distintas orientaciones, estas corresponden fundamentalmente a dos aproximaciones con diferentes visiones de la resolución de problemas: como una actividad propia de la matemática o como una tarea educativa relevante en los procesos de enseñanza y aprendizaje. Ello nos permite agrupar las investigaciones en dos grandes bloques: (a) aquellas centradas en enseñar a resolver problemas y (b) aquellas centradas en estudiar cómo pensamos cuando resolvemos problemas (Castro, 2008). Dentro de estas últimas, ubicamos los estudios centrados en los distintos estadios o fases del pensamiento por las que un resolutor o grupo de resolutores transita cuando resuelve problemas matemáticos. Nuestro trabajo se enmarca dentro de estos estudios. Como antecedentes sobre los estadios encontramos los trabajos de Artzt y Armour-Thomas (1990, 1992), Erbas y Okur (2012), Goos, Galbraith, y Renshaw (2002), Lee y Hollebrands (2006), Lester (1985), Poincaré (1908), Pólya (1945), Schoenfeld (1985), Villegas, Castro y Gutiérrez (2009), Yerushalmy (2000) o Yimer y Ellerton (2006, 2010). Antes de continuar y dado que la noción de estadio, fase o etapa admite diversos significados, precisamos que nosotros optamos por emplear el término estadio, atendiendo a la noción de episodio descrita por Schoenfeld (1985): "is a period of time during which and individual or a problem-solving group is engaged in one large task ... or a closely related body of task in the service of the same goal” (p. 292).

Los trabajos anteriormente citados, que constituyen los antecedentes de esta investigación, caracterizan los estadios de resolución de problemas matemáticos, estableciendo co- 
nexiones entre ellos y entre sus componentes. El presente estudio pretende ofrecer una orientación distinta y novedosa, que consiste en describir el proceso de resolución y cómo los estadios se interrelacionan durante dicho proceso. Para lograrlo, partimos de una metodología observacional, obtenemos datos secuenciales de la ocurrencia y tránsito entre estadios cuando parejas de resolutores resuelven un problema matemático. A dichos datos se le aplica un análisis secuencial de procesos y la técnica de coordenadas polares (Anguera y Losada, 1999; Bakeman y Gottman, 1989; Bakeman y Quera, 1996, 2011b; Gorospe y Anguera, 2000, Sackett, 1980, Sharpe y Kiperwas, 2003; Yoder y Symons, 2010), con objeto de obtener información acerca de la concurrencia entre estadios, la obtención de patrones o secuencias de estadios, frecuencias y probabilidades de transición, así como las interrelaciones globales entre estadios.

\section{Estadios en resolución de problemas}

Se puede considerar que la investigación sobre estadios en resolución de problemas científicos y matemáticos tiene su punto de partida en los trabajos de Poincaré (1908) y Dewey (1916). Estos autores, desde una visión matemática-psicológica y tomando en consideración resolutores ideales, caracterizan los distintos estadios del pensamiento cuando se resuelven problemas matemáticos. Décadas después y con una orientación cercana a la docencia y la investigación educativa, Pólya (1945) considera que el proceso de resolución implica que el resolutor tiene que superar diversos obstáculos en su camino hacia la solución del problema y que, para ello, tiene que poner en juego distintos procesos cognitivos. Aunque Pólya considera los procesos metacognitivos solo implícitamente, puso de relieve la importancia del pensamiento heurístico y del razonamiento matemático en los procesos de resolución de problemas matemáticos. El modelo de Pólya consta de cuatro estadios por los que el resolutor debe transitar sucesivamente: (a) comprender el problema, (b) concebir un plan, (c) ejecutar un plan y (d) examinar la solución. La influencia del trabajo de Pólya queda patente en numerosos trabajos. Entre ellos, están el marco para la evaluación de la resolución de problemas de PISA-2012 (Organisation for Economic Co-operation and Development [OECD], 2010, 2014), los documentos curriculares de diferentes países (Ministerio de Educación y Ciencia [MEC], 2006, 2007a, 2007b; National Council of Teacher of Mathematics [NCTM], 2000), o en diversas investigaciones (Artz y Armour-Thomas, 1990, 1992; Lee y Hollebrands, 2006; Schoenfeld, 1985, Yerushalmy, 2000; Yimer y Ellerton, 2010). 
Schoenfeld (1985), bajo la influencia del trabajo de Pólya, analiza la toma de decisiones (cognición) y las medidas de control y autocontrol (metacognición) que se ponen en juego durante la resolución de problemas matemáticos. Desde una perspectiva macroscópica, presenta un modelo de los siguientes estadios en la resolución de problemas matemáticos: (a) lectura, (b) análisis, (c) exploración, (d) planificación, (e) implementación, y (f) verificación. Schoenfeld, tomando como unidad de análisis el proceso de resolución de estudiantes trabajando individualmente, observa que el tránsito y las relaciones entre los estadios durante la resolución no es lineal, debido principalmente a la puesta en juego durante la resolución de procesos metacognitivos junto a los cognitivos. Esto marca una clara diferencia respecto a las ideas expuestas por Pólya.

Posteriormente, Artz y Armour-Thomas (1990, 1992), retomando el trabajo de Schoenfeld, se centran en los procesos cognitivos y metacognitivos puestos en juego en cada estadio. El modelo propuesto por Artz y Armour-Thomas distingue los procesos cognitivos y metacognitivos en cada estadio y considera como unidad de análisis, a diferencia de Schoenfeld, el comportamiento de parejas de estudiantes durante la resolución de problemas matemáticos. Esta unidad de análisis propicia la aparición de un estadio propio relativo al trabajo en parejas: estadio de observación y escucha. También deciden dar entidad propia de estadio a las acciones cuyo objetivo es comprender el problema. De esta forma, el modelo de Artz y Armour-Thomas está formado por los siguientes estadios: (a) lectura, (b) comprensión, (c) análisis, (d) exploración, (e) planificación, (f) implementación, (g) verificación y (h) observación y escucha. Además, el trabajo de Artz y Armour-Thomas sugiere que aquellos estudiantes que tienen éxito en los procesos de resolución describen un continuo vaivén entre los procesos cognitivos y metacognitivos, y entre los distintos estadios, tanto de los procesos internos a cada estadio por de los inter-estadios, siendo estos vaivenes más frecuentes en aquellos procesos de resolución con éxito del problema.

Yerushalmy (2000), con base en el modelo de Schoenfeld, centra su atención especialmente sobre el estadio de implementación y cómo el uso de los recursos durante el proceso de resolución crea nuevas formas de interacción entre los distintos estadios. Al igual que Artz y Armour-Thomas (1992), adopta como unidad de análisis parejas de estudiantes resolviendo problemas de álgebra, desde una aproximación funcional y utilizando tecnología digital. Yerushalmy detecta vaivenes entre pequeños periodos en el estadio de planificación, con fuerte presencia de procesos metacognitivos, muchos de los cuales son motivados por la in- 
clusión de tecnología digital, y grandes periodos en el estadio de implementación. El autor sugiere que la cantidad de vaivenes entre estadios necesita ser explorada con mayor profundidad, con objeto de comprender mejor como se desarrollan o evolucionan las estrategias y la planificación en la resolución de problemas matemáticos. Motivados por dicha sugerencia, Lee y Hollebrands (2006) estudian los procesos de resolución de problemas presentados en formato de pequeño programa informático que contiene un applet java específicamente diseñado. Para ello, proponen problemas a estudiantes de educación primaria y adaptan los indicadores de cada estadio, añadiendo uno nuevo: organización. Lee y Hollebrands consideran un modelo de seis estadios: (a) análisis, (b) planificación, (c) implementación, (d) evaluación, (e) verificación y (f) organización. Estos autores detectan que en aquellos estudiantes que logran resolver más frecuentemente los problemas, la secuencia de estadios "implementación>evaluación->implementación” es también más frecuente. Sin embargo, aquellos estudiantes que tienen menos éxito, presentan menos procesos de control tras periodos de tiempo en el estadio de implementación. Además, señalan que el uso de los applets es generalmente empleado en el estadio de implementación y casi no es usado en los estadios de análisis y planificación.

Yimer y Ellerton $(2006,2010)$ desarrollan un modelo de cinco estadios: (a) comprometer, (b) transformar-formular, (c) ejecutar, (d) evaluar y (e) internalizar, para describir la variedad de transiciones durante los procesos de resolución de problemas. Los autores confirman las observaciones de Schoenfled, al detectar cómo las transiciones entre los cinco estadios no son lineales ni unidireccionales. Yimer y Ellerton añaden que estas transiciones usualmente involucran un periodo de relectura que sirve como "catalizadores de las decisiones metacognitivas que se producen y orientan a la elección del camino a seguir o la puesta en juego de otras acciones metacognitivas dentro del mismo estadio en el que los estudiantes se encuentran" (p. 251). Kursat y Okur (2012) obtienen similares conclusiones, añadiendo que para que los resolutores tengan éxito, deben conocer cuándo y cómo usar las estrategias en cada episodio, conociendo las habilidades metacognitivas y transiciones que deben poner en juego.

Estudios anteriores emplean distintos modelos de estadios en la resolución de problemas y distintas unidades de observación (sujetos individuales o parejas). Un elemento común en todos es la utilización de la técnica pensamiento verbal en voz alta (Ericsson y Simon, 1980, 1993) para el registro del proceso de resolución. Dicho método ha permitido a los in- 
vestigadores analizar las verbalizaciones y acciones que explícitamente llevan a cabo los resolutores, y también analizar aquellas inferidas a través del habla, generando un fuerte incremento en la cantidad de procesos que pueden ser observados si los comparamos con aquellos producidos en condiciones de silencio (Villegas et al. 2009). La técnica del pensamiento en voz alta no es única de las investigaciones en resolución de problemas. Por ejemplo, Armengol (2007) la utiliza para describir los estadios en el proceso del aprendizaje de la escritura. También ha sido empleada en estudios de autocontrol y autoregulación de parejas de resolutores (Goos et al., 2002) o el análisis de patrones de representación en la resolución de problemas de optimización (Villegas et al., 2009). Parece razonable entonces que consideremos utilizar esta técnica para el registro del proceso de resolución.

\section{Análisis secuencial y coordenadas polares}

Los antecedentes presentados en el epígrafe anterior constatan el esfuerzo y avance de la comunidad de investigadores en Educación Matemática en la identificación y caracterización de los distintos estadios en resolución de problemas, el tránsito entre ellos, las relaciones intra e inter estadios y el análisis de las componentes cognitivas y metacognitivas de ello. Aunque las investigaciones señaladas anteriormente suponen un avance en la comprensión de los procesos de resolución, los autores coinciden en señalar la necesidad de continuar indagando sobre los estadios y el proceso de resolución de problemas matemáticos. Haciendo nuestra dicha necesidad, planteamos la utilización de la metodología observacional (Anguera, 1990, 2003; Riba, 1993) para obtener probabilidades de causalidad, concurrencia, patrones e interrelaciones de los procesos objeto de observación, en nuestro caso de los estadios en la resolución de problemas matemáticos. Una ventaja de utilizar este enfoque metodológico es que brinda la posibilidad de complementar tratamientos cualitativos y cuantitativos (Anguera, 2010; Riba, 1993). Esto supone un elemento diferenciador respecto a trabajos anteriores. Sometemos la observación del proceso de resolución, a través de la técnica del pensamiento en voz alta (Ericsson y Simon, 1980, 1993), a un determinado registro mediante el apoyo de un sistema de codificación o sistema de categorías compuesto por los distintos estadios en resolución de problemas y que describimos más adelante. Dicho sistema permite la obtención de datos secuenciales a los que aplicar procedimientos observacionales para un tratamiento cuantitativo de la observación. Entre los procedimientos observacionales de corte cuantitativo, destacan aquellos relativos al análisis secuencial de procesos (Bakeman y Gottman, 1989; Bakeman y Quera, 1996, 2011b; Sharpe y Koperwas, 2003; Yoder y Symons, 2010). Este tipo 
de análisis nos permite separarnos de los modelos descriptivos y exploratorios tradicionalmente empleados en las investigaciones en resolución de problemas matemáticos y considerar: (a) el estudio de concurrencias y de relaciones-patrones secuenciales entre los distintos estadios y en distintos retardos o lag [ $\operatorname{lag} 0$ indica concurrencia, lag 1, indica siguiente, lag -1 indica antecesor,...] a través de las probabilidades condicionadas, frecuencias de transición y puntuaciones $z$ (residuos ajustados normalizados) con nivel de significación $\alpha<.05$; (b) la exploración de interrelaciones globales entre estadios a través de la técnica de coordenadas polares (Sackett, 1980) en su variante de retroactividad genuina (Anguera y Losada, 1999; Gorospe y Anguera, 2000). Según estos autores, las coordenadas polares posibilitan objetivar en qué medida cada categoría (en nuestro caso, cada estadio del proceso de resolución) estimula o inhibe a las demás categorías (estadios). Aplicar dicha técnica constituye un elemento innovador en el estudio de las interrelaciones entre los estadios en la resolución de problemas matemáticos.

En la literatura científica, el análisis secuencial de procesos se ha empleado en estudios psicológicos. Por ejemplo, Symons, Hoch, Dahl y McComas (2003) lo emplean para estudiar la relación entre el comportamiento autodestructivo y la conducta comunicativa en personas. García (1993) utiliza el análisis secuencial de procesos para estudiar la relación entre el desarrollo cognitivo y el comunicativo de niños autistas, en ambos casos, la unidad de análisis son sujetos individuales. En cambio, otros trabajos emplean el análisis secuencial de procesos cuando el centro de interés está relacionado con la interacción social. Así, Gimeno, Anguera, Berzosa y Ramírez (2006) lo usan para estudiar las relaciones en el estilo de comunicación en familias con hijos adolescentes. El empleo del análisis secuencial permitió, por ejemplo, obtener patrones de secuencias entre el comportamiento autodestructivo y la conducta comunicativa que lo estimulaba, o la existencia de interacción simétricas entre la familia y el hijo adolescente o que el estilo de comunicación está en función del género del adolescente.

Dado que el análisis secuencial y, en especial, la técnica de coordenadas polares son relativamente recientes $y$, aunque originariamente parte de la psicología, su potencialidad para obtener de patrones y relaciones entre observables no ha pasado inadvertido para otros campos científicos. Especialmente interesante es aplicarlo cuando el núcleo de interés tiene relación con procesos de interacción social, como muestran los estudios en el ámbito de la Educación Física y Deportiva. Por ejemplo, Castellano (2000) y Perea (2008) analizan las relaciones entre el comportamiento de jugadores de un equipo de fútbol y las acciones de juego; Goros- 
poe y Anguera (2000), Gorospe, Hernández, Anguera, y Martínez (2005) centrados en el tenis; o Cayero (2008) respecto del voleibol. Recientemente, la investigación educativa comienza a presentar trabajos que emplean el análisis secuencial de procesos. Así, Kapur (2011) lo utiliza para la obtención de patrones de interacción entre un grupo de estudiantes que trabajan colaborativamente en la resolución de problemas de física a través del chat, o Liao, Chen, Cheng y Chan (2012) para el estudio de conductas de aprendizaje en interacción puestas en juego por grupos de estudiantes en ambientes de aprendizaje basados en juegos. La potencialidad del análisis secuencial va más allá de los estudios que involucran a sujetos. Por ejemplo, Jiménez y Perales (2000) lo utilizan para detectar los patrones o guiones didácticos subyacentes en los textos escritos e ilustraciones de libros de texto de física y química en educación secundaria.

Como hemos señalado previamente, la aplicación de la técnica de coordenadas polares, en su variante de retrospectividad genuina (Anguera y Losada, 1999; Gorospe y Anguera, 2000), es un elemento innovador dentro de los estudios sobre las interrelaciones entre los estadios en la resolución de problemas matemáticos. La principal razón es que dicha técnica nos permite analizar las interrelaciones globales de estimulación e inhibición entre las categorías de observación que, en nuestro caso, serán los distintos estadios en resolución de problemas.

Supongamos que disponemos de un sistema de estadios (categorías) exhaustivo y mutuamente excluyente compuesto por los estadios $\{$ A, B, C, D, E $\}$. Dicha técnica consiste en fijar un estadio E, y considerar: (a) la perspectiva prospectiva considerando las puntuaciones $z$ en el número de retardos lag positivos considerados, tomando E como estadio criterio o dado y los demás (A B C D) como objetivo; y (b) la perspectiva retrospectiva, análoga pero considerando ahora lag o retardos negativos (Figura 1).

Las puntuaciones $z$ se obtienen utilizando una versión estandarizada del residuo propuesto por Haberman (1979, citado en Bakeman y Quera, 2011a):

$$
Z_{i j}=\frac{\left(X_{i j}-M_{j j}\right)}{\sqrt{M_{i j} \times\left(1-\frac{X_{i j}}{X_{+j}}\right) \times\left(1-\frac{X_{j j}}{X_{j+}}\right)}}
$$

Donde 
- $\mathrm{X}_{\mathrm{jj}}=$ frecuencia observada para la celda $i j$ que indica el número de ocurrencias de la

transición entre el estadio $i$ y el estadio $j$ en el lag o retardo considerado. Por ejemplo, dada la secuencia $\mathrm{ABCAB}$, para lag+1 (siguiente); $\mathrm{X}_{11}=0 ; \mathrm{X}_{12}=2 \mathrm{o}_{13}=0$ tomando filas y columnas

$(\mathrm{A}, \mathrm{B}, \mathrm{C})$.

$-\mathrm{X}_{+\mathrm{j}} \mathrm{y} \mathrm{X}_{\mathrm{j}+}$ frecuencias observadas por columnas y filas.

$-\mathrm{X}_{++}$frecuencia observada total.

- $\mathrm{M}_{\mathrm{jj}}=\frac{\mathrm{x}_{\mathrm{i}+} \times \mathrm{x}_{+\mathrm{j}}}{\mathrm{x}_{++}}$es la frecuencia esperada para la celda $i j$.

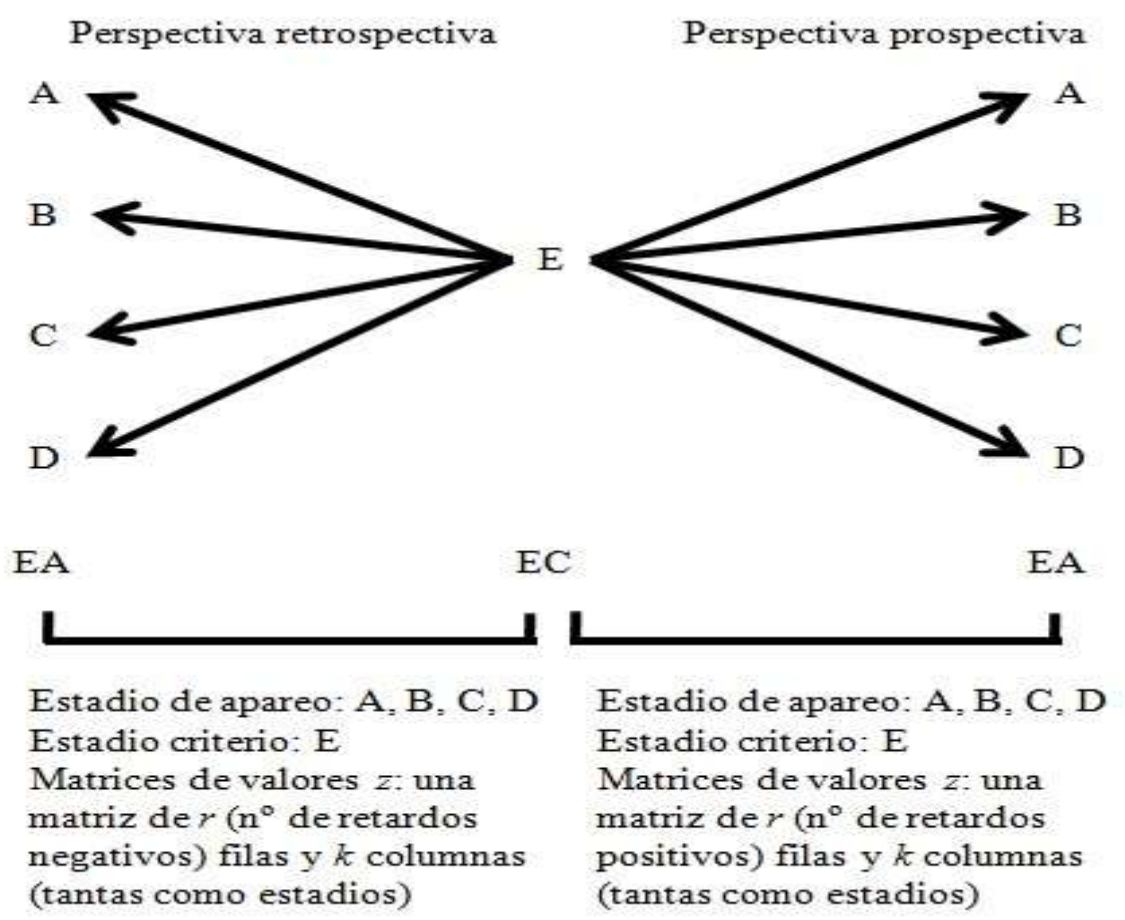

Figura 1. Adaptación del modelo de coordenadas polares (Gorospe y Anguera, 2000, p. 279)

Partiendo entonces de las puntuaciones $z$ en los respectivos retardos, se calculan los valores del estadístico $Z_{\text {sum }}=\frac{2 z}{\sqrt{n}}$ siendo $n$ el número de retardos considerados (Sackett, 1980). Según este autor, el estadístico $Z_{\text {sum }}$ es un potente reductor de datos que conlleva una elevada capacidad informativa de las relaciones entre los estadios. La variante de la retrospectividad genuina introduce el cálculo del estadístico para los retardos positivos $(\mathrm{de}+1 \mathrm{a}+5)$ en 
la perspectiva prospectiva $\left[Z_{\text {sumX }}\right]$ y retardos negativos (de -1 a -5$)$ en la retrospectiva $\left[Z_{\text {sumY }}\right]$. Anguera y Losada (1999) y Gorospe y Anguera (2000) consideran el par ( $\left.Z_{\text {sumX }}, Z_{\text {sumY }}\right)$ como las coordenadas que permiten definir vectores con origen el centro de coordenadas $(0,0)$. La representación de tales vectores en un sistema coordenado proporciona un mapa de relaciones de inhibición y estimulación entre estadios. Dado que se utilizan las puntuaciones $z$, las relaciones serán significativas cuando los módulos de los vectores son superiores a 1.96, mientras que el ángulo respecto del origen informa acerca de la naturaleza (estimulación o inhibición) de la relación. Sintetizamos la interpretación de los cuadrantes de las coordenadas polares en la Figura 2.

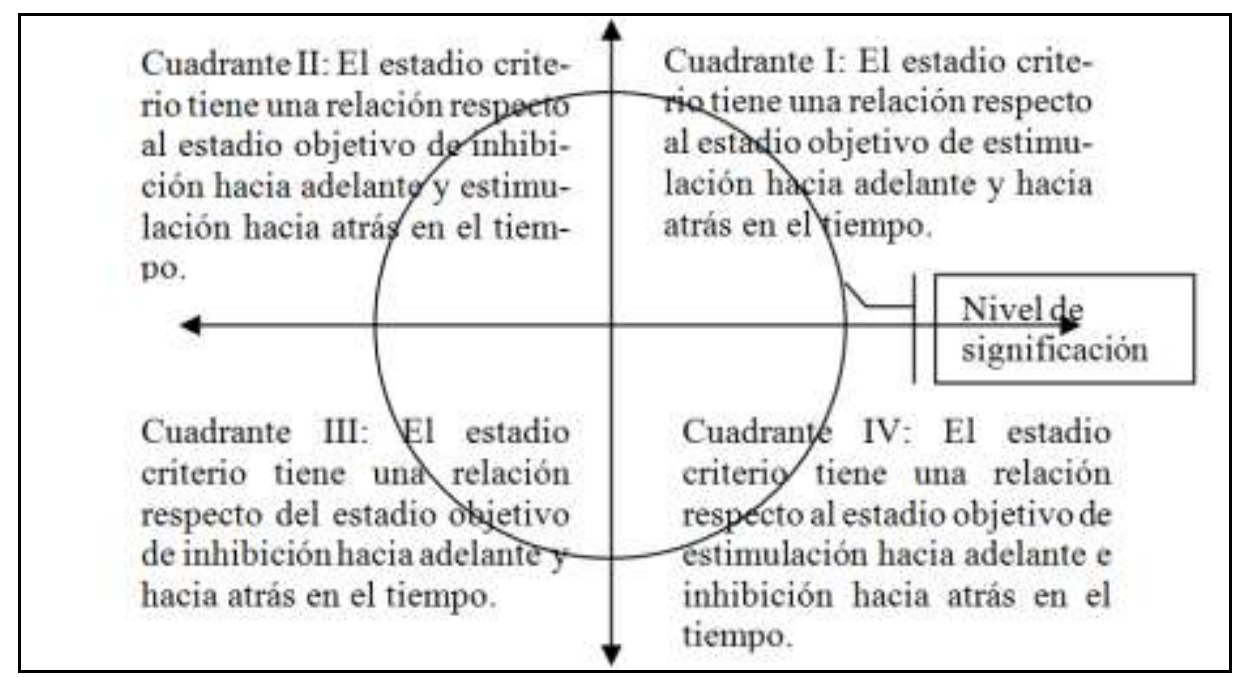

Figura 2. Interpretación cuadrantes de las coordenadas polares

Objetivo e hipótesis

En este trabajo nos planteamos dos hipótesis en relación a la resolución por parejas de problemas matemáticos. Por un lado, la existencia de patrones y relaciones de causalidad entre los distintos estadios en la resolución de problemas matemáticos, es decir, la existencia de relaciones secuenciales entre estadios. Y por el otro, que es posible objetivar cómo cada estadio estimula o inhibe a los demás estadios en el proceso de resolución de problemas matemáticos, es decir, la existencia de interrelaciones globales entre estadios. Así, nuestro objetivo es:

Estudio de las relaciones secuenciales e interrelaciones globales entre los distintos estadios en la resolución de problemas matemáticos. 


\section{Método}

\section{Participantes}

Dado el carácter innovador y descriptivo de este estudio, llevamos a cabo el análisis del proceso de resolución de problemas de una pareja de estudiantes de un problema matemático. En este caso, utilizamos un problema de optimización del tipo cálculo de distancias (Camacho y González, 1998). La pareja estaba compuesta por dos estudiantes universitarios de la Licenciatura de Matemáticas, de sexo femenino, y con 22 y 23 años de edad, respectivamente. La elección de los sujetos (A1 y A2) es intencional de entre aquellos estudiantes que se presentaron voluntariamente a la experiencia. Los motivos principales para la elección de estos estudiantes es que estaban habituados al trabajo en parejas y al trabajo colaborativo, a hacer uso de tecnología digital, a resolver problemas matemáticos y a argumentar, explicar y discutir su proceso de resolución.

\section{Procedimiento}

El espacio donde trabajaron los sujetos fue un seminario con la distribución de elementos y medios técnicos que mostramos en la Figura 3, i.e., dos cámaras de video, una grabadora de audio, una televisión de 32 pulgadas, un ordenador portátil con el enunciado del problema a resolver y un cañón proyector. Una de las cámaras enfocaba a los sujetos y estaba conectada con la televisión. La otra cámara estaba enfocada a la proyección de las imágenes del ordenador portátil a través del proyector. La televisión permitió, por un lado que el observador visualizara las acciones de los sujetos en primer plano; y, por otro lado, ocultarlo del campo de visión de los sujetos. La grabadora situada cerca de la pareja permite recoger aquellas verbalizaciones que se realizan en voz baja, minimizando la pérdida de información. 


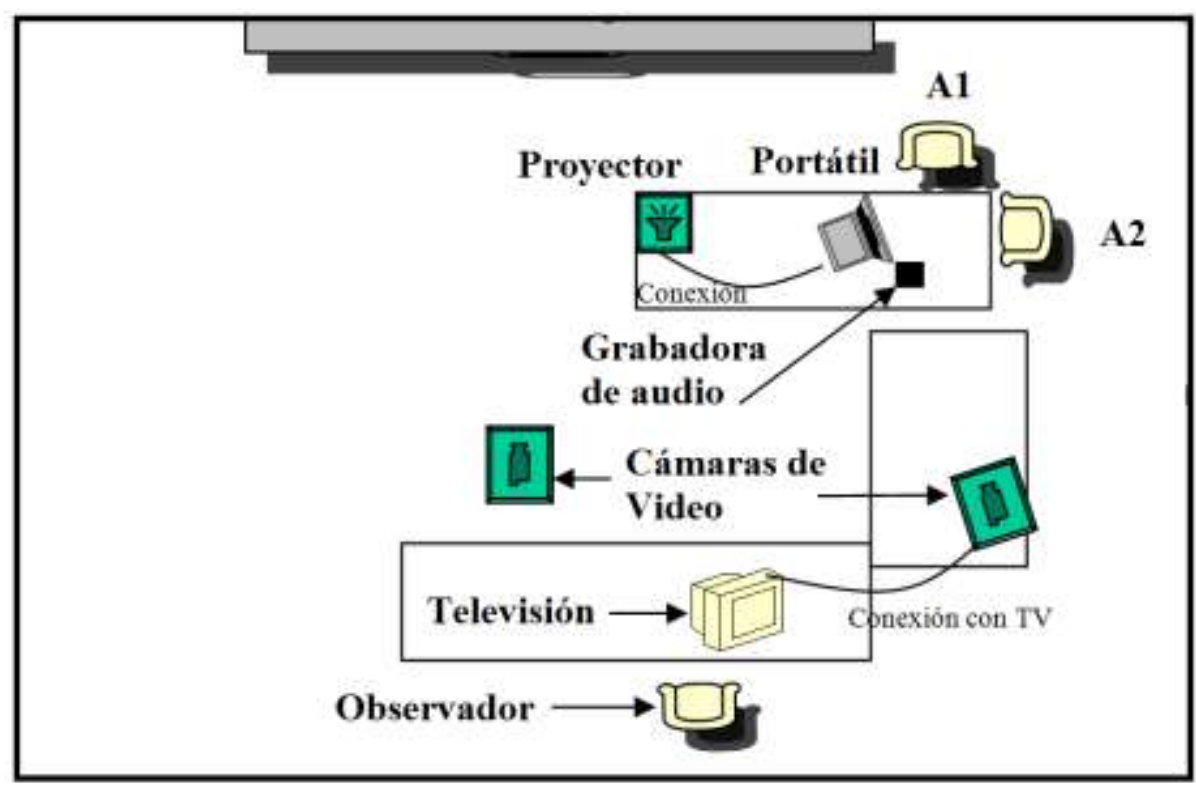

Figura 3. Espacio de trabajo y medios técnicos

Los sujetos contaron con 30 minutos para la resolución del problema propuesto. $\mathrm{Pu}$ dieron utilizar el ordenador y hojas de papel para la resolución y los invitamos a que emplearan la técnica del pensamiento en voz alta. La infraestructura diseñada permitió obtener dos videograbaciones, una audiograbación y dos registros escritos de notas in situ, uno del observador y otro de la pareja.

Las videograbaciones son sincronizadas y superpuestas utilizando la técnica del CromaKey y el software de edición de video Studio 9.0 (Avid Technology, 2005). Obtuvimos entonces dos nuevos registros audiovisuales, uno con los sujetos como imagen principal y otro con las acciones-reacciones llevadas a cabo en el portátil como imagen secundaria. Transcribimos el audio a través del software libre Subtitle Workshop 2.51 (Gluskin, 2004). Eventualmente, para intervalos de audio de baja calidad, utilizamos la audiograbación. Finalmente sincronizamos la transcripción a los videos utilizando el software libre VirtualDub 1.9.11 (Lee, 2010) y el filtro subtitler.vdf (Figura 4). El proceso puesto en juego consigue minimizar el sesgo asociado a la pérdida de información tanto visual como auditiva, y proporciona dos registros que capturan la actuación de los sujetos durante la resolución del problema. 


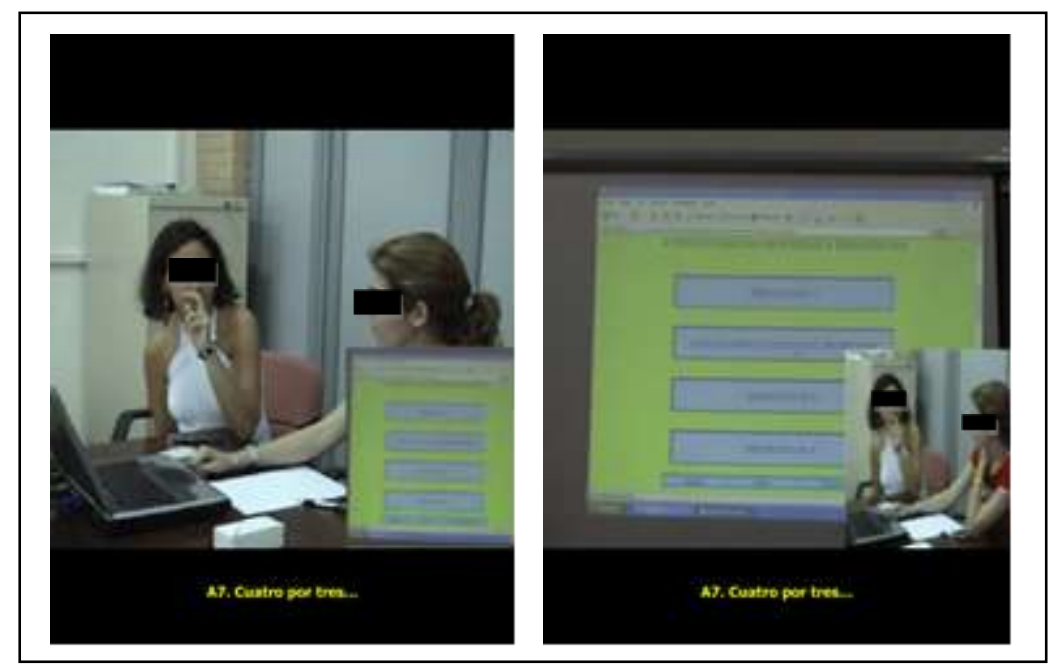

Figura 4. Videos superpuestos con transcripción del audio en formato subtítulo

\section{Problema matemático propuesto}

El problema matemático propuesto es un problema de optimización [Figura 5] integrado en un diseño web a modo de i-Actividad: “Actividades en formato web cuyo objetivo es facilitar el desarrollo de la propia actividad y el consiguiente aprendizaje a través de la interactividad del ordenador con el estudiante" (Codina, Cañadas y Castro, 2011, p. 159). La iActividad consiste en un conjunto de 6 páginas entrelazadas con instrucciones, anuncios, sugerencias y pequeños applets con los que la pareja puede interactuar, diseñada para guiar y facilitar el tránsito entre estadios y el proceso de resolución del problema. Por otro lado, la elección de esta tipología de problema cuyo enunciado está descrito en la Figura 5, está motivada por: (a) están presentes en la actividad humana de manera natural con una gran variedad de aplicaciones y situaciones cotidianas que facilita la activación de la experiencia previa e intuición en los resolutores (Malaspina, 2007); (b) permiten distintas posibilidades de representación y modelización que posibilita la puesta en juego de una diversidad de métodos, estrategias y conexiones (Villegas et al., 2009); (c) los avances tecnológicos, especialmente el desarrollo de software educativo, facilitan su resolución y tratamiento en el aula (Camacho y González, 1998; Forbes, 2001; Scher, 1999; Verderber, 1992) y; (d) son objeto de aprendizaje en las matemáticas escolares (González y Sierra, 2004; Malaspina, 2011; MEC, 2006, 2007a, 2007b; NCTM, 2000). 


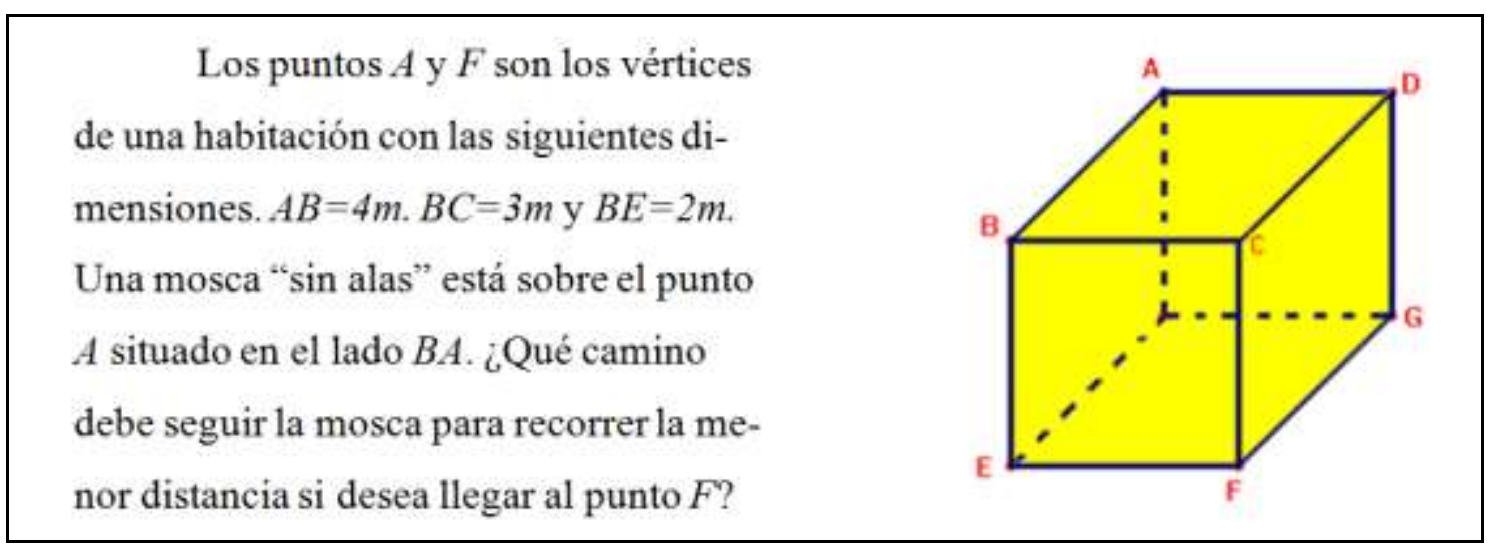

Figura 5. Enunciado del problema de la i-Actividad

\section{Instrumento}

Para la recogida de datos contextualizados, exhaustivos y objetivos de los procesos relacionados con los estadios en la resolución de problemas matemáticos elaboramos un instrumento de observación ad hoc a través de un sistema mixto, que combina el formato campo y los sistemas de categorías (Bakeman y Gottman, 1989). Partiendo de un sistema germinal basado en los trabajos Pólya (1945), Schoenfeld (1985) y Artzt y Armour-Thomas (1990, 1992), elaboramos un primer instrumento de observación, que fue sometido a reajustes y modificaciones en función de observaciones no sistemáticas llevadas a cabo en tres experiencias piloto previas. Dichos reajustes han tenido en cuenta tanto las especificidades derivadas de la influencia de la interactividad de la i-Actividad así como del trabajo en parejas. Finalmente, el instrumento de observación está compuesto por ocho estadios exhaustivos y mutuamente excluyentes (cada estadio solo puede asignarse a un proceso observado), que constituyen las categorías empleadas para la codificación de la información:

(a) Lectura (Lec): El sujeto realiza la lectura del enunciado e interioriza las condiciones y objetivo del problema.

(b) Análisis (Ana): El sujeto "in analysis an attempt is made to fully understand a problem, to select an appropriate perspective and reformulate the problem in those terms, and to introduce for consideration whatever principles or mechanisms might be appropriate" (Original) (Schoenfeld, 1985, p. 298). El sujeto "considers domain-specific knowledge that is relevant to the problem" (Artz y Armour-Thomas, 1992, p.172). 
(c) Exploración (Exp): El sujeto emplea estrategias entendidas como procedimientos o reglas que permiten responder a cuestiones haciendo uso de relaciones y conceptos de una estructura conceptual (Rico, 1997). Idealmente el sujeto no tiene un procedimiento estructurado de acción y necesita ejercer mayor control sobre su progreso a través de evaluaciones locales y globales. En cierto sentido es una revisión de la estructura del problema en busca de información relevante que se pueda incorporar a una secuencia análisis-plan-ejecución.

(d) Planificación (Pla): El sujeto selecciona los pasos y las estrategias que, potencialmente, pueden conducir a la resolución del problema.

(e) Implementación (Imp): El sujeto ejecuta acciones previamente estructuradas en la planificación.

(f) Verificación (Ver): El sujeto lleva a cabo una evaluación o control local durante el proceso de resolución del problema así como cuando realiza una evaluación global de la resolución y solución. Por tanto, esta etapa puede ser transversal a las anteriores.

(g) Observación y escucha (Obs): El sujeto parece estar atendiendo y observando el trabajo del compañero. Este estadio puede ocurrir con mayor frecuencia con estudiantes que trabajan en grupo.

(h) Conversación (Con): El sujeto está manteniendo una conversación con el compañero aparentemente ajena al proceso de resolución o bien realiza algún comentario al docenteobservador.

Al considerar los estadios como "a period of time during which and individual or a problem-solving group is engaged in one large task ... or a closely related body of task in the service of the same goal" (Schoenfeld, 1985, p. 292), el instrumento de observación permite el registro de la ocurrencia o no de los distintos estadios en un único flujo de comportamiento secuencial. Utilizando el software Atlas-ti 6.0 (Muhr, 2010), obtenemos un conjunto de datos (códigos) secuenciales con tiempo, que posteriormente son transformados al formato del software específico para el análisis secuencial GSEQ 5.1.07 (Bakeman y Quera, 2011a). Dicho flujo transformado describe el proceso de resolución en función de la ocurrencia, dura- 
ción y transiciones entre los distintos estadios, a través de códigos de tal forma que podemos implementar los análisis estadísticos disponibles en el software software GSEQ.

\section{Fiabilidad y validez}

El flujo de datos obtenido es continuo, presentando constancia intrasesional (no se presentaron circunstancias excepcionales de ruptura de la actividad durante la sesión). Los datos no tienen sesgo por inobservabilidad, el existente es de tipo tecnológico y no supera el 5\% del tiempo total de observación como recomiendan (Anguera, 1990; Hernández, 1996). Los estadios son activados por transiciones y registrados únicamente en los instantes en los que se produce la transición entre ocurrencias (Bakeman y Gottman, 1989).

Dado que se dispone de las grabaciones de la sesión, utilizamos la técnica de concordancia consensuada para garantizar la fiabilidad intraobservador al lograr el acuerdo entre observadores antes del registro de datos (Anguera, 1990; 2003). En cuanto a la fiabilidad interobservador, comparamos la codificación del $15 \%$ de la duración total de la sesión, elegida al azar y codificada a través de concordancia consensuada por tres observadores, con la codificación del mismo periodo por otro observador. Siguiendo la recomendación propuesta por Bakeman y Quera (2011b), obtenemos los índices Kappa por unidad de tiempo con tolerancia \pm 2 segundos; por alineamiento de eventos con tolerancia 5 y solapamiento del $80 \%$, el Kappa máximo y el porcentaje de acuerdo. La Tabla 1 muestra los valores obtenidos que según Landis y Koch (1977, tomado de Bakeman y Quera, 2011b) son excelentes.

Tabla 1. Fiabilidad interobservadores

\begin{tabular}{|c|c|c|c|c|c|}
\hline \multicolumn{3}{|c|}{ Tolerancia \pm 2 sg } & \multicolumn{3}{|c|}{ Tolerancia 5, solapamiento $80 \%$} \\
\hline Por unidad de & Kappa & & Por & Kappa & \\
\hline tiempo & Máximo & Acuerdo & Alineamiento & Máximo & Acuerdo \\
\hline $.84-.86$ & $.89-.89$ & $88 \%-89 \%$ & .76 & .88 & $82 \%$ \\
\hline
\end{tabular}

Por otro lado, para garantizarnos que los distintos estadios presentan algún tipo de asociación en los distintos retardos o lag, aplicamos de la prueba Chi-Cuadrado a nivel de significación $\mathrm{p}<.01$. Los resultados informan de la existencia de asociaciones salvo quizás para el retardo o lag 5 y -5 (Tabla 2 ). 
Tabla 2. Chi-Cuadrado por retardos o lag

\begin{tabular}{|c|c|c|}
\hline Retardos & $\chi^{2}$ & gl \\
\hline lag -5 & 47.27 & 49 \\
\hline $\operatorname{lag}-4$ & 82.63 & $49 *$ \\
\hline lag -3 & 81.56 & $49 *$ \\
\hline $\operatorname{lag}-2$ & 138.43 & $49 *$ \\
\hline lag -1 & 88.83 & $41^{*}$ \\
\hline $\operatorname{lag} 1$ & 88.83 & $41 *$ \\
\hline $\operatorname{lag} 2$ & 138.43 & $49 *$ \\
\hline lag 3 & 81.56 & $49 *$ \\
\hline $\operatorname{lag} 4$ & 82.63 & $49 *$ \\
\hline $\operatorname{lag} 5$ & 47.27 & 49 \\
\hline
\end{tabular}

Diseño y análisis de datos

Propusiomos un diseño para el análisis de los datos a dos niveles de especificidad: (a) para cada sujeto o por sujetos y (b) para la pareja (P). Para cada nivel se obtienen:

1) Un conjunto de estadísticas descriptivas básicas para cada estadio: frecuencia, duración, probabilidad simple y duración media (Tabla 3).

Tabla 3. Estadísticas básicas, estudiante A1, A2 y pareja

\begin{tabular}{|c|c|c|c|c|c|c|c|c|c|c|c|c|}
\hline \multirow[b]{2}{*}{ Estadio } & \multicolumn{3}{|c|}{ Frecuencia } & \multicolumn{3}{|c|}{ Duración } & \multicolumn{3}{|c|}{ Probabilidad } & \multicolumn{3}{|c|}{$\begin{array}{c}\text { Duración } \\
\text { Media }\end{array}$} \\
\hline & A1 & A2 & $\mathrm{P}$ & A1 & $\mathrm{A} 2$ & $\bar{P}$ & A1 & A2 & $\bar{P}$ & A1 & A2 & $P$ \\
\hline Lec & 19 & 13 & 32 & 204 & 178 & 382 & .2 & .2 & .2 & 11.0 & 14.0 & 12.0 \\
\hline Ana & 13 & 17 & 30 & 96 & 157 & 253 & .1 & .1 & .1 & 7.4 & 9.2 & 8.4 \\
\hline Exp & 18 & 7 & 25 & 215 & 58 & 273 & .2 & .1 & .1 & 12.0 & 8.3 & 11.0 \\
\hline Pla & 1 & 0 & 1 & 13 & 0 & 13 & .0 & .0 & .0 & 13.0 & 0.0 & 13.0 \\
\hline Imp & 7 & 10 & 17 & 189 & 82 & 271 & .2 & .1 & .1 & 27.0 & 8.2 & 16.0 \\
\hline Ver & 24 & 21 & 45 & 177 & 184 & 361 & .2 & .2 & .2 & 7.4 & 8.8 & 8.0 \\
\hline Con & 18 & 7 & 25 & 46 & 26 & 72 & .0 & .0 & .0 & 2.6 & 3.7 & 2.9 \\
\hline
\end{tabular}




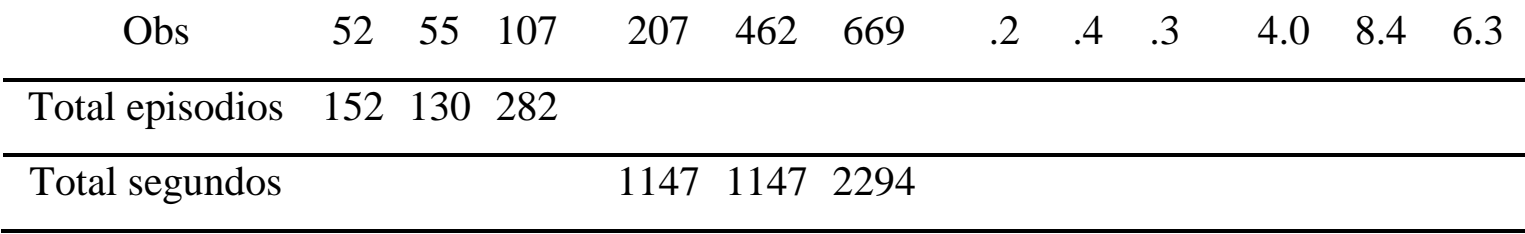

2) Los períodos de trabajo en paralelo: intervalos de tiempo $\left(t_{n}, t_{m}\right)$ con $m>n$ en el que la actuación de los estudiantes es codificada en estadios distintos del criterio resolución de problemas; y de trabajo colaborativo: intervalos de tiempo $\left(\mathrm{t}_{\mathrm{n}}, \mathrm{t}_{\mathrm{m}}\right)$ con $\mathrm{m}>\mathrm{n}$ en el que la actuación de los estudiantes es codificada en el mismo estadio del criterio resolución de problemas (Tabla 4). Es decir, durante el trabajo colaborativo, ambos sujetos centraron su atención en aspectos de la resolución del problema que se encuadran en el mismo estadio; mientras que en el trabajo en paralelo, centraron su atención es aspectos no necesariamente complementarios de distintos estadios del proceso de resolución. La información que se extrae del estudio de los periodos de trabajo en paralelo y del trabajo colaborativo permite describir cómo la pareja abordó conjuntamente la resolución del problema.

Tabla 4. Coocurrencias entre estadios de A1 y A2

\begin{tabular}{|c|c|c|c|c|c|c|c|c|c|c|c|c|c|c|c|c|c|c|}
\hline & & eec & $\mathrm{A} 2$ & Ana & $\mathrm{A} 2$ & & A2 & & $\mathrm{A} 2$ & & $\mathrm{~A} 2$ & Ver & $\mathrm{A} 2 \mathrm{C}$ & Con & $\mathrm{A} 2 \mathrm{C}$ & & To & tal \\
\hline & $\mathrm{fr}$ & $\mathrm{T}$ & fr & $\mathrm{T}$ & fr & T & $\mathrm{fr}$ & $\mathrm{T}$ & fr & $\mathrm{T}$ & fr & $\mathrm{T}$ & fr & $\mathrm{T}$ & fr & $\mathrm{T}$ & fr & $\mathrm{T}$ \\
\hline A1Lec & 11 & 149 & 1 & 6 & 1 & 6 & 0 & 0 & 0 & 0 & 2 & 12 & 0 & 0 & 8 & 31 & 23 & 204 \\
\hline A1Ana & 1 & 1 & 7 & 51 & 0 & 0 & 0 & 0 & 1 & 2 & 2 & 27 & 0 & 0 & 6 & 15 & 17 & 96 \\
\hline A1Exp & 5 & 15 & 3 & 7 & 7 & 22 & 0 & 0 & 0 & 0 & 2 & 4 & 2 & 11 & 20 & 156 & 39 & 215 \\
\hline A1Pla & 0 & 0 & 1 & 4 & 0 & 0 & 0 & 0 & 0 & 0 & 0 & 0 & 0 & 0 & 0 & 9 & 1 & 13 \\
\hline A1Imp & 1 & 2 & 0 & 0 & 0 & 0 & 0 & 0 & 9 & 75 & 6 & 48 & 0 & 0 & 14 & 64 & 30 & 189 \\
\hline A1Ver & 1 & 2 & 7 & 42 & 2 & 8 & 0 & 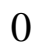 & 0 & 0 & 13 & 66 & 0 & 0 & 18 & 59 & 41 & 177 \\
\hline A1Con & 1 & 1 & 0 & 0 & 0 & 0 & 0 & 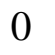 & 0 & 0 & 0 & 0 & 4 & 8 & 13 & 37 & 18 & 46 \\
\hline $\mathrm{A} 1 \mathrm{Obs}$ & 3 & 8 & 9 & 47 & 4 & 22 & 0 & 0 & 3 & 5 & 9 & 27 & 4 & 7 & 28 & 91 & 60 & 207 \\
\hline Total & 23 & 178 & 28 & 157 & 14 & 58 & 0 & 0 & 13 & 82 & 34 & 184 & 10 & 26 & 107 & 462 & 229 & 1147 \\
\hline
\end{tabular}

Nota: fr=frecuencia; $\mathrm{T}=$ tiempo medido en segundos. En cursiva frecuencia y tiempo de trabajo colaborativo.

3) Los grafos de probabilidades de transición entre estadios, los cuales informan acerca de la probabilidad de que un estadio suceda en orden de ocurrencia a otro (mostramos solo las probabilidades superiores a .2), y el conjunto de cadenas diádicas y triádicas significativas a nivel $\alpha<.05$. Dado que no se disponen de datos suficientes para la utilización de las proba- 
bilidades condicionadas, se utiliza las tablas de p-valores obtenidos con el software PSEQ de análisis de permutaciones (Bakeman, Robinson y Quera, 1996). Las cadenas, a nivel $\alpha<.05$ se conforman considerando en cada retardo o lag aquellas relaciones significativas de igual dirección (estimulación o inhibición) y teniendo en cuenta que: (a) no se cumple la propiedad transitiva, y (b) se termina cuando existe una doble, triple..., bifurcación o existen dos retardos sin relaciones significativas (max-lag) (Figura 6).

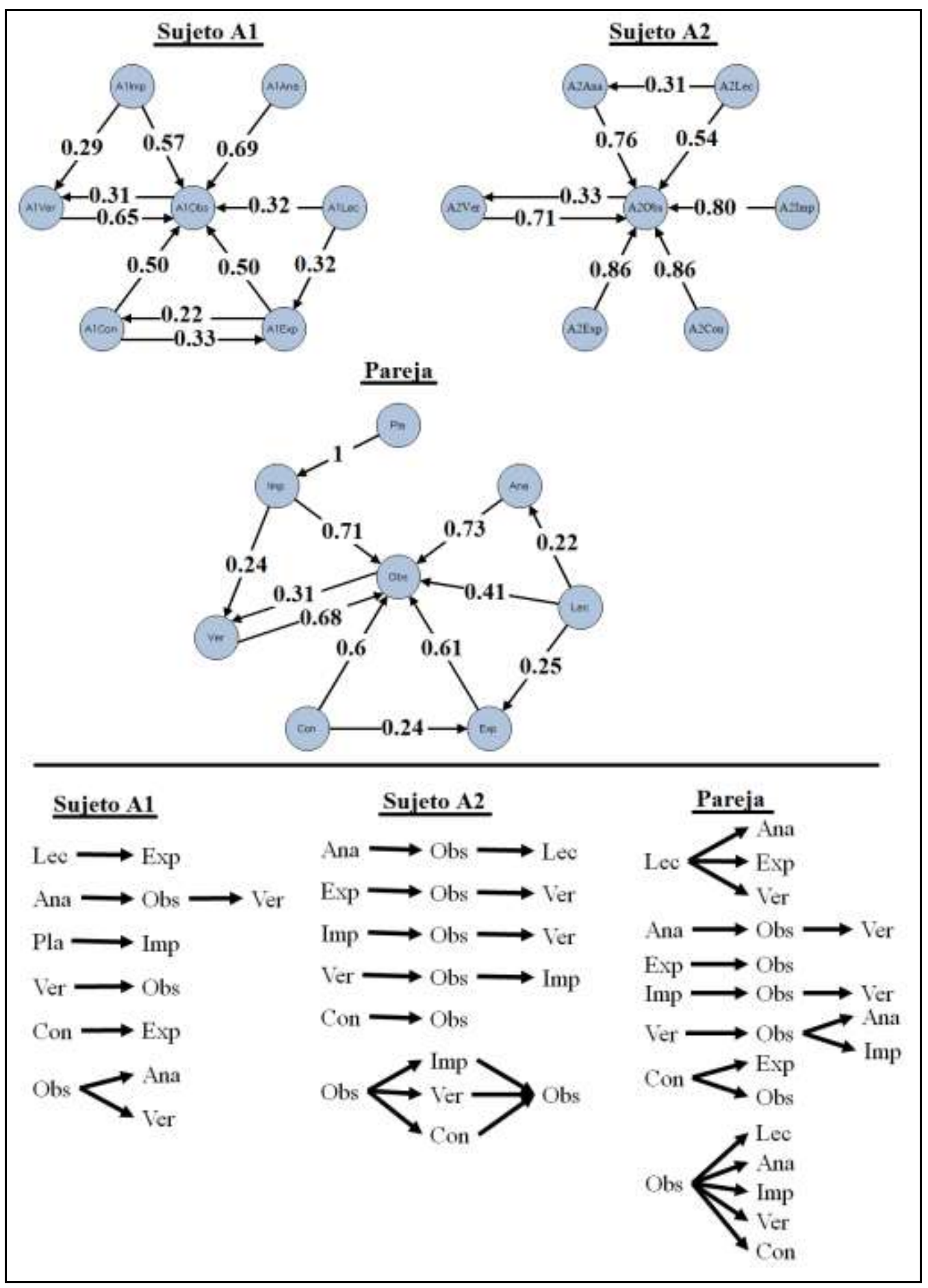

Figura 6. Grafos de transición y cadenas significativas entre estadios 
4- Los mapas de interrelaciones globales entre estadios. Aplicamos la técnica de coordenadas polares sobre las puntuaciones $z$ para los retardos -5 a 5 y nivel $\alpha<.05$ (Figuras $7 \mathrm{a}$, $7 b, 7 c)$.

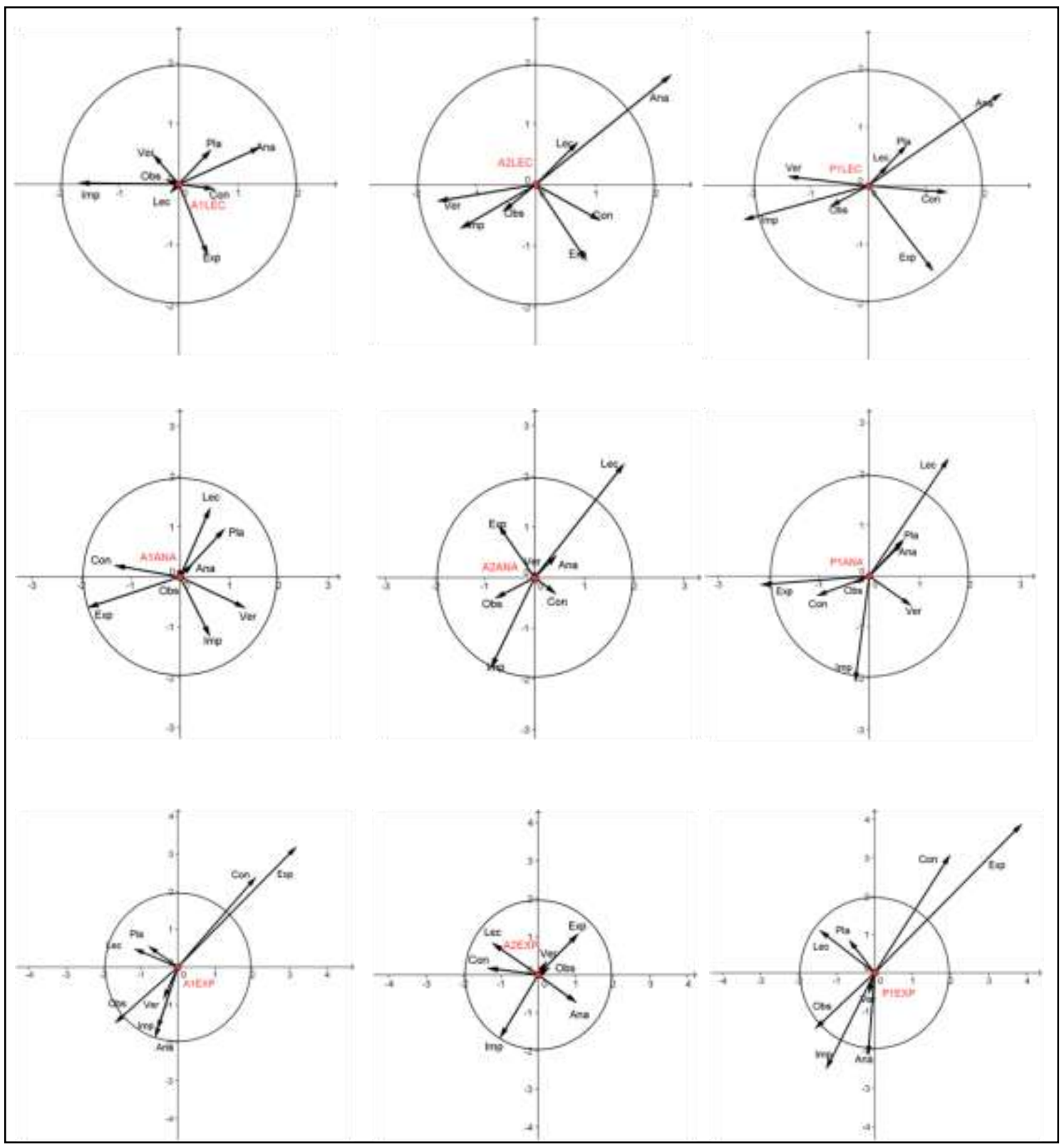

Figura 7a. Gráfico de coordenadas polares, estadios Lectura, Análisis y Exploración

A modo de ejemplo, veamos cómo interpretar uno de estos mapas. El mapa de relaciones con conducta criterio exploración para la pareja (Figura 7a) nos informa de la existencia una relación de estimulación entre los estadios exploración y conversación, y una autoestimulación de la categoría exploración en ambas componentes, la prospectiva y la retrospectiva. 
Ello quiere decir que, más allá de la ocurrencia debida al azar, en la pareja hay periodos de exploración que anteceden y preceden no muy alejado en orden de ocurrencia a periodos de conversación, y viceversa. También quiere decir que exploración se autoantecede y autoprecede. Similar razonamiento se realiza para las tres relaciones obtenidas de inhibición en ambas componentes, en concreto entre exploración y observación y escucha; entre exploración e implementación; y entre exploración y análisis.

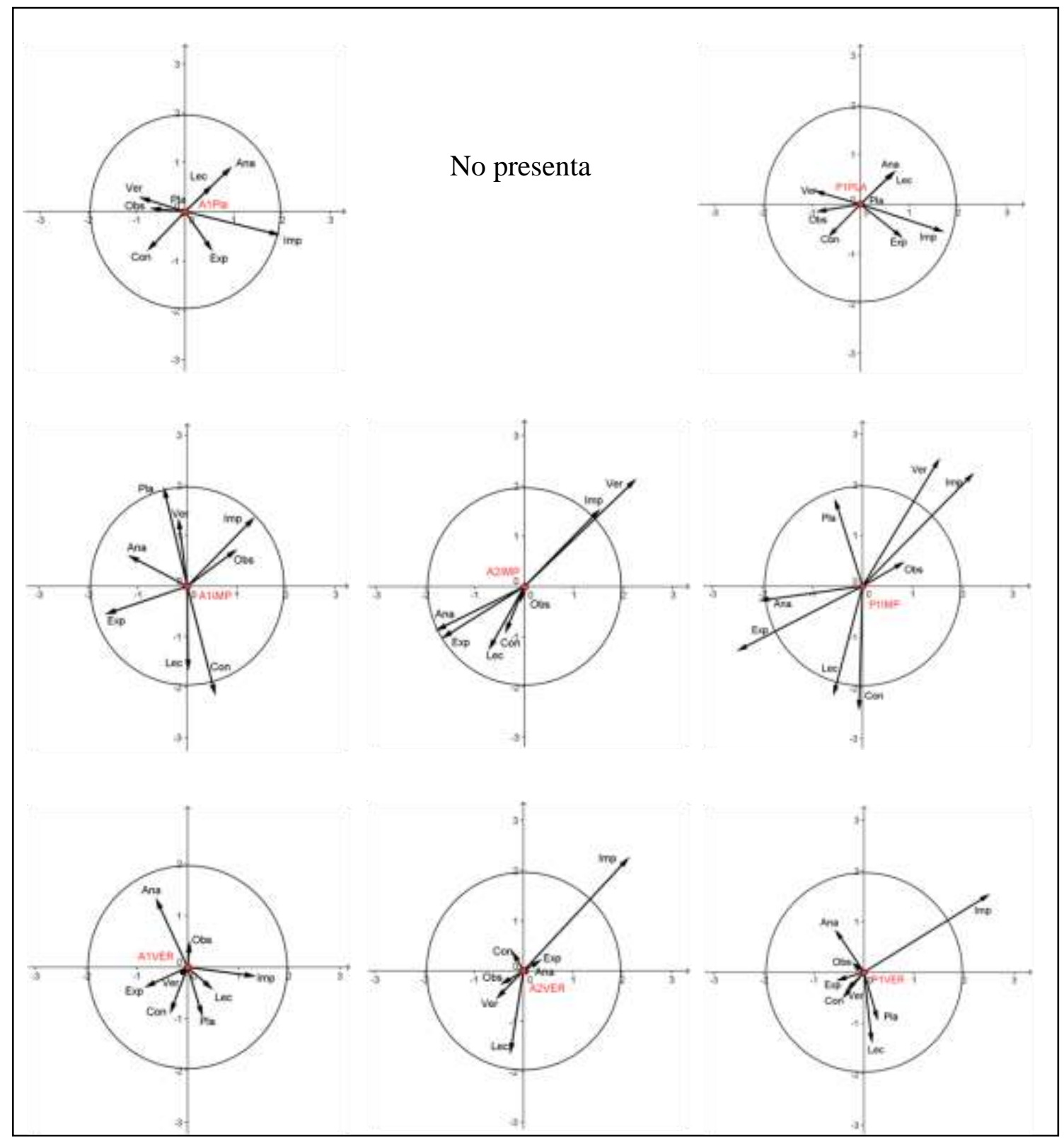

Figura $7 b$. Gráfico de coordenadas polares, estadios Planificación, Implementación y Verificación 


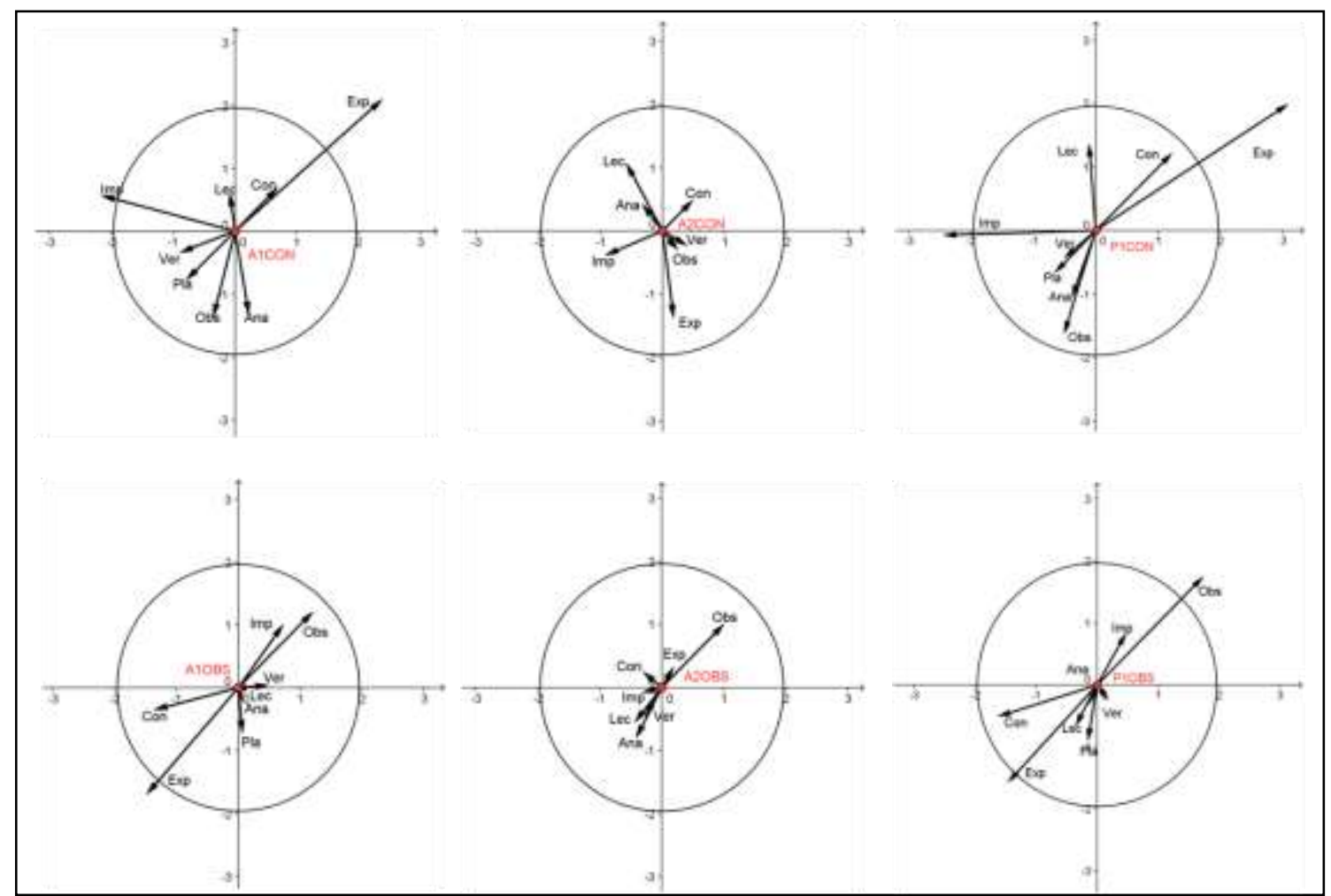

Figura 7c. Gráfico de coordenadas polares, estadios Conversación y Observación y Escucha

5) Las traslaciones de ejecución: aquellas situaciones en las que al menos uno de los estudiantes de la pareja, en un periodo determinado, es codificado en el estadio del compañero/as en el periodo inmediatamente anterior. La existencia de dichas relaciones se deriva de la existencia de autoestimulaciones y autoinhibiciones surgidas del análisis de coordenadas polares. Su estudio constituye un elemento innovador de indagación en el campo de la resolución de problemas matemáticos en parejas (Tabla 5).

Se han definido tres tipologías de traslaciones de ejecución para dos sujetos (A y B):

a) Traslación de continuidad de ejecución de A: cuando si en un periodo de tiempo $\left(\mathrm{t}_{\mathrm{n}}\right.$, $t_{m}$ ) el sujeto $B$ está en el estadio $X$, entonces en el periodo inmediatamente siguiente el sujeto A está en el estadio X. Admite con dos posibilidades para B, que mantenga el estadio $\mathrm{X}$ o que no lo mantenga y se le asigne en dicho periodo otro estadio Y diferente.

b) Traslación de continuidad de ejecución de B: igual que traslación de continuidad de ejecución de A intercambiando B por A y A por B. 
c) Traslación mutua de ejecución: cuando en un periodo de tiempo $\left(t_{n}, t_{m}\right)$, si el sujeto A está en el estadio X y B en Y, entonces en el periodo inmediatamente siguiente, A está en el estadio Y y B en X (intercambio de estadios).

Tabla 5. Traslación de Ejecución entre A1 y A2

\begin{tabular}{|c|c|c|c|c|c|c|}
\hline Tipo & A1 & $\mathrm{A} 2$ & SigA1 ${ }^{a)}$ & $\operatorname{SigA} 2^{\text {b) }}$ & Frec & Total \\
\hline \multirow{4}{*}{ Mutua } & Ana & Obs & Obs & Ana & 1 & \\
\hline & Obs & Exp & Exp & Obs & 1 & \\
\hline & Obs & Ver & Ver & Obs & 1 & \\
\hline & Obs & Con & Con & Obs & 1 & 4 \\
\hline \multirow[t]{16}{*}{ A1->A2 A2 Mantiene } & Exp & Obs & Obs & Obs & 6 & \\
\hline & Con & Obs & Obs & Obs & 6 & \\
\hline & Obs & Ver & Ver & Ver & 5 & \\
\hline & Ver & Obs & Obs & Obs & 3 & \\
\hline & Lec & Obs & Obs & Obs & 2 & \\
\hline & $\operatorname{Imp}$ & Obs & Obs & Obs & 2 & \\
\hline & Obs & Ana & Ana & Ana & 2 & \\
\hline & Obs & $\operatorname{Imp}$ & $\operatorname{Imp}$ & $\operatorname{Imp}$ & 2 & \\
\hline & Lec & Ana & Ana & Ana & 1 & \\
\hline & Exp & Con & Con & Con & 1 & \\
\hline & Ana & Obs & Obs & Obs & 1 & \\
\hline & Ana & Lec & Lec & Lec & 1 & \\
\hline & Exp & Ana & Ana & Ana & 1 & \\
\hline & Obs & $\operatorname{Exp}$ & $\operatorname{Exp}$ & $\operatorname{Exp}$ & 1 & \\
\hline & Obs & Lec & Lec & Lec & 1 & \\
\hline & Obs & Con & Con & Con & 1 & 36 \\
\hline \multirow[t]{7}{*}{ A2 No Mantiene } & Exp & Con & Con & Obs & 1 & \\
\hline & Imp & Obs & Obs & Con & 1 & \\
\hline & Ver & Obs & Obs & Con & 1 & \\
\hline & Ana & Obs & Obs & Ver & 1 & \\
\hline & $\operatorname{Imp}$ & Obs & Obs & Ver & 1 & \\
\hline & Ver & Obs & Obs & $\operatorname{Imp}$ & 1 & \\
\hline & Obs & $\operatorname{Imp}$ & Imp & Ver & 1 & 7 \\
\hline
\end{tabular}




\begin{tabular}{lllllll} 
A2->A1 A1 Mantiene & Imp & Obs & Imp & Imp & 7 & \\
& Ver & Obs & Ver & Ver & 5 & \\
& Exp & Obs & Exp & Exp & 4 & \\
& Exp & Lec & Exp & Exp & 2 & \\
& Obs & Ver & Obs & Obs & 2 & \\
& Obs & Ana & Obs & Obs & 1 & \\
& Ver & Ana & Ver & Ver & 1 & \\
& Ana & Obs & Ana & Ana & 1 & \\
& Obs & Con & Obs & Obs & 1 & 24 \\
\hline A1 No mantiene & Obs & Ana & Ver & Obs & 3 & \\
& Obs & Con & Ana & Obs & 1 & \\
Ver & Obs & Imp & Ver & 1 & 5 \\
\hline b) SigA1= estadio siguiente para A1 & & & & & \\
${ }^{\text {b) estadio siguiente para A2 }}$ & & & & &
\end{tabular}

\section{Resultados}

La pareja compuesta por los sujetos A1 y A2 consiguen resolver el problema. El tiempo total invertido es de 1147 segundos (19 minutos y 17 segundos). Se registran un total de 282 periodos de tiempo o eventos para la pareja. De ellos, 152 se corresponden a A1 y 130 a A2. El estadio observación y escucha está fuertemente presente durante el proceso de resolución con una frecuencia de 107 eventos, duración total 669 s, duración media 6.03 s y probabilidad .3. De estos 107 eventos, 52 corresponden a A1 y 55 a A2, mientras que la duración total de observación y escucha para A2 dobla la duración total de A1 (462 s y 207 s respectivamente). Ello permite sugerir que es A1 quien lleva el peso de la resolución. Esta deducción se refuerza por el hecho de que: (a) en el estadio de exploración, A1 dobla en frecuencia de evento y duración total a A2 (18 vs 7 eventos; 215 s vs 58 s respectivamente); (b) A1 realiza más procesos de lectura que A2 (19 vs 13 eventos; 204 s vs 178 s respectivamente); (c) A1 aunque realiza menos procesos de implementación en frecuencia que A2 (7 vs 10 eventos), duplica en duración total al tiempo de A2 (182 s vs 82 s); (d) solo A1 presenta un evento de la categoría Planificación, con una duración de 13 s, y (e) A2 realiza más procesos de análisis que A1 (17 vs 13 eventos; 157 s vs 96 s respectivamente). Por último, aunque a priori pareciera que la lógica nos sugiere que el estadio de verificación debería de haber sido más frecuente 
y con una duración mayor para A2, que mantenga cierta equivalencia en ambos nos revela el intento de la pareja por crear y mantener un espacio de resolución conjunto del problema (Roschelle y Teasley, 1995), monitorizando continuamente ambos estudiantes su proceso de resolución.

En cuanto a las concurrencias entre estadios (Tabla 4), los resultados muestran que los estudiantes han trabajado colaborativamente cerca del $40 \%$ del tiempo total (462 s de $1147 \mathrm{~s}$ ), representando el 35\% del total de eventos registrados (79 de 229 eventos). De los 462 s, 149 s se corresponden con el estadio lectura, lo cual es natural máxime cuando al inicio del proceso de resolución, ambos estudiantes invierten su tiempo en la lectura y comprensión del enunciado del problema. Similar razonamiento explica los 91 s correspondientes al estadio observación y escucha puesto que cuando se produce una interacción con alguno de los applet de la iActividad, ambos suelen observar la pantalla del ordenador. De los restantes estadios, cabe resaltar que durante 51 s A1 y A2 están simultáneamente en el estadio de análisis, correspondiendo respectivamente al 53\% y 32\% del tiempo total de dicho estadio (96 s y 157 s respectivamente). Durante este periodo los estudiantes intentan analizar y seleccionar una perspectiva que les permita abordar la resolución con éxito del problema. Asimismo durante $75 \mathrm{~s}$ ambos estudiantes simultáneamente realizan procesos propios del estadio de implementación, correspondiendo respectivamente al 40\% para A1 y el 92\% para A2 del tiempo total del estadio (189 s y 82 s respectivamente). Nótese que A2 realiza tareas de implementación de manera independiente solo el $8 \%$ del tiempo. En cuanto al estadio de exploración, los estudiantes tuvieron dificultades para seleccionar perspectivas comunes con las que explorar el problema o bien, cada uno realizó las exploraciones de manera independiente. Prueba de ello son los 22 s en los que A1 y A2 están simultáneamente en el estadio exploración, representando solo el $10 \%$ del tiempo total de exploración para A1 (215 s.) mientras que representa el $40 \%$ para A2 (58 s). Por último, los dos están simultáneamente realizando tareas de verificación el 37\% del tiempo total de dicho estadio (66 s de 177 s y 184 s).

Fijamos nuestra atención ahora en el tránsito por los distintos estadios para lo que elaboramos los grafos de transición y de transiciones significativas entre estadios diádicas y triádicas (Figura 6). Con los grafos de transición, tanto a nivel de sujetos como de pareja, el estadio observación y escucha está fuertemente relacionada con los demás estadios, incluso con probabilidades cercanas a .8. También la Figura 6 muestra como las únicas probabilidades mayores a .2 en ambas direcciones se producen entre los estadios verificación y observación y 
escucha. Ello sugiere que los estudiantes una vez realizada alguna evaluación local observan la reacción que ésta produce en el compañero/a, y viceversa, la observación produce la aparición de evaluaciones locales. De hecho, la transición diádica Obs $\rightarrow$ Ver es significativa en los dos niveles (por sujetos y por pareja). Por otro lado, las probabilidades de transición entre las categorías conversación y exploración, junto con las cadenas diádicas significativas Con $\rightarrow$ Exp para A1, y Con $\rightarrow$ Obs para el sujeto A2 vuelve a sugerir que durante el estadio exploración, la pareja no ha estado coordinada o no han realizado un trabajo colaborativo.

Por otro lado, la existencia de probabilidades superiores a .2 entre los estadios implementación y verificación en los dos niveles junto con la existencia de la cadena significativa triádica Imp $\rightarrow$ Obs $\rightarrow$ Ver para la pareja y para A2, indica que la pareja ha intentado controlar y minimizar los posibles errores de cálculo durante el estadio de implementación, es especial el sujeto A2. Por último, que la cadena diádica Lec $\rightarrow$ Exp sea significativa para A1 y la pareja así como que en todas las cadenas de A2 interviene la categoría observación y escucha refuerza el hecho de que es A1 quien señala la dirección en el proceso de resolución mientras que A2 ejerce de supervisor de dicho proceso.

El análisis de resultados realizado hasta ahora se ha puesto en juego un máximo de dos retardos, el empleo de la técnica de coordenadas polares nos permite ir más allá al informar acerca de la estimulación e inhibición entre los estadios, tanto en la componente prospectiva como en la retrospectiva sin especificar el retardo, o lo que es lo mismo, desde una visión global del proceso. Es decir, los mapas de relaciones que se detallan en las Figuras 7a, 7b y 7c van más allá del estudio de los eventos siguientes y por tanto, nos permiten relacionar los comportamientos de los sujetos más allá de las situaciones instantáneas o inmediatamente anterior/posterior. En la medida de lo posible elaboramos nuestra narración en base a las relaciones de estimulación por un lado y las relaciones de inhibición por el otro.

Comencemos por las relaciones de estimulación. El estadio de lectura estimula en las componentes prospectivas y retrospectivas al estadio de análisis para A2 mientras que para A1 no se presenta dicha relación. En cambio, para la pareja, lectura y análisis se estimulan mutuamente en ambas componentes. Es decir como pareja tras un proceso de lectura, proceden al análisis de la información y a su vez, cuando dicho análisis genera dudas, vuelven a la 
lectura. A nivel de pareja intentan disponer de una comprensión profunda del problema, potenciada especialmente por A2.

Los estadios exploración y conversación se estimulan mutuamente en las componentes prospectivas y retrospectivas para A1, mientras que para A2 no se presenta dicha relación. En cambio, nuevamente a nivel de pareja, ambos estadios se estimulan mutuamente en ambas componentes. Es decir, como pareja y durante la exploración del problema recurren a pequeños "descansos" que pueden facilitar la toma de consciencia del proceso de resolución. Dichos descansos están potenciados por A1 que, como se ha venido observando en los análisis previos, es quien dirige la resolución del problema. Por otro lado, a nivel de pareja, exploración se autoestimula lo que implica que la pareja ejerce control sobre su progreso y busca información que pueda ser incorporada a la resolución del problema. Este hecho en relación a los resultados expuestos anteriormente pone de manifiesto cómo actuaciones independientes, en mayor o menor medida, conllevan una visión coordinada de las actuaciones a nivel de pareja al contrario de lo que se podría esperar.

El estadio planificación estimula a Implementación en la componente prospectiva y lo inhibe en la restrospectiva solo para A1. Ello es lógico, al ser solo A1 quien establece un plan de resolución que posteriormente es llevado a cabo y sobre el que A1 no presenta dudas al no detectarse estimulaciones en la componente retrospectiva.

Los estadios implementación y verificación se estimulan mutuamente en ambas componentes para A2, mientras que para A1 no se presenta dicha relación. Pero a nivel de pareja, los estadios implementación y verificación se estimulan mutuamente en ambas componentes. Es decir, a nivel de pareja se ejerce control sobre la ejecución del plan de resolución, llevándose a cabo especialmente por el sujeto A2. Por otro lado que el estadio implementación estimule en la componente retrospectiva al estadio conversación para A1, nos informa que éste realiza pequeños descansos durante la ejecución del proceso de resolución. Ello explica a su vez las relaciones de autoestimulación de implementación tanto para A2 como para la pareja.

Por último, que el estadio observación y escucha se autoestimula en ambas componentes a nivel de pareja es coherente con el funcionamiento de un proceso de resolución colaborativo por parejas pues indica que ambos sujetos observan las acciones del compañero constantemente. 
En cuanto a las relaciones de inhibición, que los estadios lectura e implementación se inhiban mutuamente en los dos niveles es coherente con un proceso de resolución en el que existe un plan de resolución afianzado, lo contrario indicaría que se ha realizado una lectura superficial del problema y que se ejecutan acciones sin un plan preestablecido coherente.

Los estadios análisis y exploración se inhiben en ambas componentes para A1, ello sugiere que este sujeto ha alcanzado una comprensión una comprensión de la situación problemática que posteriormente le permite diseñar un plan de resolución en el que confía. Por su parte, A2 asume dicho plan ya que a nivel de pareja también obtenemos dicha relación de inhibición. Por otro lado la existencia de inhibición mutua de los estadios análisis e implementación para A2 y la pareja es coherente con un proceso de resolución en el que se dispone de un plan de resolución estructurado previo a la implementación. Todo ello a su vez explica las relaciones de inhibición mutua en ambas componentes de los estadios exploración y observación y escucha para A1 y la pareja, y entre los estadios exploración e implementación para A2 y la pareja. Por último, que el estadio de Implementación inhiba al estadio de planificación y conversación en la componente prospectiva para A1 muestra la seguridad que este sujeto tiene en su plan de resolución.

Por último, en cuanto a las traslaciones de ejecución (Tabla 5) los resultados muestran que todas involucran al estadio observación y escucha indicando la existencia de continuidad doble en los estadios involucrados. Por otro lado, de las 43 traslaciones de ejecución para el sujeto A1, A2 mantiene su estadio en 36 ocasiones. Ello nos informa que existen 36 periodos de tiempo de trabajo colaborativo motivados por la acción de A2 en los que A1 intenta seguir o mantener los planteamientos-sugerencias que A2 realiza, cabe destacar las 5 ocasiones que involucra al estadio verificación (A1 valida también las observaciones realizadas por A2) y en 4 al estadio análisis (A1 continúa con las acciones realizadas por A2). Respecto a las traslaciones de A1 hacía A2 en las que A2 no mantiene su categoría, la mayoría involucra la categoría observación.

Inversamente se recogen 29 traslaciones de ejecución para el sujeto A2. De ellas A1 mantiene el estadio en 24 periodos, es decir, se producen 24 periodos más de trabajo colaborativo. A diferencia de las traslaciones inversas descritas anteriormente, en esta ocasión las traslaciones de A2 hacía el estadio de A1 no involucran mayoritariamente al estadio observa- 
ción y escucha, siendo las que más frecuencia presenta aquellas que implican al estadio implementación, el estadio verificación y el estadio de exploración. Quiere decir esto que A2 colabora en la implementación del plan de resolución propuesto por A1, que A2 necesita explorar el problema a diferencia de A1 y que A2 ejerce de revisor del proceso de resolución.

\section{Discusión y concluciones}

La investigación sobre los estadios en resolución de problemas matemáticos se ha centrado tradicionalmente en la descripción del tránsito entre estadios o en el análisis de sus componentes. El presente trabajo pone de manifiesto que el empleo del análisis secuencial y la técnica de coordenadas polares permite describir el tránsito entre estadios, la obtención de patrones de transición y relaciones de causalidad entre estadios, así como la obtención de las interrelaciones de estimulación e inhibición globales entre estadios significativas acaecidas durante el proceso de resolución de un problema matemático. Nuestro trabajo responde afirmativamente a nuestras hipótesis y es original en cuanto al método y técnica, abriendo nuevas posibilidades de indagación no solo en los estadios en resolución de problemas, sino en el propio campo de la Educación Matemática y por extensión, sobre cualquier actividad escolar en la que existan o puedan existir relaciones de causalidad secuencial.

Para lograr explorar las posibilidades del análisis secuencial elaboramos un instrumento de observación que incorpora el estadio conversación como un nuevo elemento a tener en cuenta en el estudio de la resolución de problemas matemáticos por parejas. Como hemos puesto de manifiesto, dicho estadio ha posibilitado establecer la influencia de esos pequeños periodos conversación en el proceso de resolución de las parejas, especialmente en los estadios de exploración e implementación. El estadio conversación, para la pareja, ejerce de puente entre la exploración del problema y los procesos de implementación.

A su vez, hemos llevado a cabo un análisis en distintos niveles de concreción. Por un lado, cuando consideramos como unidad de análisis al conjunto de acciones puestas de manifiesto por ambos sujetos (nivel de pareja) y por el otro, a las acciones de cada uno de los sujetos (nivel sujetos). Dichos niveles de concreción han permitido la obtención de resultados que describen las influencias individuales en las acciones de la pareja como muestra el hecho de la existencia de la transición triádica Imp $\rightarrow$ Obs $\rightarrow$ Ver para la pareja y para A2 y por tanto, es A2 quien más controla y minimiza el proceso de implementación de la pareja. La posibilidad de 
establecer distintas unidades de análisis conlleva que si se dispone de un número suficiente de sujetos de observación (parejas), es posible considerar la macro unidad genérica que considera al conjunto de acciones puestas de manifiesto por todos los sujetos. Dicha macro unidad entonces permitirá obtener resultados globales que pueden ser considerados en cierta medida independiente del sujeto, abriendo una nueva vía de interpretación en la resolución de problemas matemáticos.

Con la obtención de las interrelaciones significativas de estimulación e inhibición junto con las transiciones diádicas y tríadicas entre estadios, describimos el comportamiento de los sujetos y de la pareja durante el proceso de resolución. Las transiciones diádicas o triádicas nos han informado acerca de ciertas regularidades en el modo de proceder de los sujetos, mientras que las interrelaciones de estimulación e inhibición nos han informado de la naturaleza e intensidad de las relaciones entre estadios durante el proceso de resolución.

De este modo, si se reitera el procedimiento de análisis para una diversidad de situaciones, contextos y problemas matemáticos para un sujeto (o grupos de sujetos), el docente o investigador tendrán la posibilidad de identificar aquellos aspectos o estadios de la resolución de problemas en las que se presentan ciertas dificultades, y por tanto disponer de nueva información para proponer medidas específicas de aprendizaje para la resolución de problemas matemáticos. Por último, las técnicas del análisis secuencial se han mostrado como unas potentes herramientas para la descripción del trabajo colaborativo puesto en juego por los sujetos durante la resolución del problema. En particular, el estudio de las concurrencias entre estadios de los sujetos nos ha permitido la obtención de las traslaciones de ejecución, y con ello, concretar con mayor profundidad en las interrelaciones surgidas entre los sujetos durante su trabajo colaborativo.

\section{Referencias}

Anguera, M. T. (1990). Metodología observacional. En J. Arnau, M. T. Anguera y J. Gómez (Eds.), Metodología de la investigación en ciencias del comportamiento (pp. 125-236). Murcia: Universidad de Murcia.

Anguera, M. T. (2003). La observación. En C. Moreno (Ed.), Evaluación psicológica. Concepto, proceso y aplicación en las áreas del desarrollo y de la inteligencia (pp. 271308). Madrid: Sánz y Tórres. 
Anguera, M. T. (2010). Posibilidades y relevancia de la observación sistemática por el profesional de la psicología. Papeles del psicólogo, 31(1), 122-130.

Anguera, M. T. y Losada, J. L. (1999). Reducción de datos en marcos de conducta mediante la técnica de coordenadas polares. En M. T. Anguera (Ed.), Observación de conducta interactiva en contextos naturales: aplicaciones (pp. 163-188). Barcelona: Universidad de Barcelona.

Armengol, L. (2007). Los protocolos de pensamiento en voz alta como instrumento para analizar el proceso de escritura. Revista Española de Lingüística Aplicada, 20, 27-36.

Artzt, A. F., y Armour-Thomas, E. (1990, Abril). Protocol analysis of group problem solving in mathematics: a cognitive-metacognitive framework for assessment. Ponencia presentada en el Annual Meeting of the American Educational Research Association, Boston.

Artzt, A. F., y Armour-Thomas, E. (1992). Development of a cognitive-metacognitive framework for protocol analysis of mathematical problem solving in small groups. Cognition and Instruction, 9(2), 137-175. doi:10.1207/s1532690xci0902

Avid Technology (2005). Pinnacle Studio (Versión 9.0) [Software de ordenador].

Bakeman, R., y Gottman, J. M. (1989). Observación de la interacción: Introducción al análisis secuencial. Madrid: Morata.

Bakeman, R., y Quera, V. (1996). Análisis de la interacción. Madrid: Ra-Ma.

Bakeman, R., Robinson, B. F., y Quera, V. (1996). Testing sequential association: estimating exact p values using sampled permutations. Psychological methods, 1(1), 4-15.

Bakeman, R., y Quera, V. (2011a). GSEQ (Versión 5.1) [Software de ordenador]. Barcelona. Obtenido de http://www2.gsu.edu/ psyrab/gseq/index.html

Bakeman, R., y Quera, V. (2011b). Sequential analysis and observational methods for the behavioral sciences. New York: Cambridge University Press.

Camacho, M., y González, A. (1998). Una aproximación a los problemas de optimización en libros de texto de bachillerato y su resolución con la TI-92. Aula, 10, 137-152.

Castellano, J. (2000). Observación y análisis de la acción de juego en el Fútbol. (Tesis Doctoral no publicada). Vitoria-Gasteiz: Universidad del País Vasco.

Castro, E. (2008). Resolución de problemas: Ideas, tendencias e influencias en España. En G. Luengo (ed.), Investigación en Educación Matemática XII, actas del XII simposio de la Sociedad Española de Investigación en Educación Matemática. Badajoz, España: Sociedad Española de Investigación en Educación Matemática. 
Cayero, R. (2008). Observación de la acción del juego del voleibol: análisis secuencial y variabilidad. (Tesis Doctoral no publicada). Málaga: Universidad de Málaga.

Codina, A., Cañadas, M. C., y Castro, E. (2010). Diseño de una e-actividad orientada a la resolución de problemas de matemáticas. En F. Albuquerque, G. Lobato, J. F. De Matos, I. Chagas y E. Cruz (eds.), I Encontro internacional tic e educaÇão. InovaÇão curricular com TIC (pp. 1-7). Lisboa, Portugal: Instituto de EducaÇao da Universidade de Lisboa.

Codina, A., Castro, E., y Cañadas, M. C. (2011). Un sistema de categorías para el análisis de la interactividad en una i-actividad de resolución de problemas. En J. L. Lupiañez, M. C. Cañadas, M. Molina, M. Palarea, M. y A. Maz (eds.), Investigaciones en pensamiento numérico y algebraico e historia de la matemática en educación matemática (pp. ). Granada: Departamento de Didáctica de la Matemática. Universidad de Granada.

Dewey, J. (1916). Essays in experimental logic. Chigaco: University of Chicago Press

Dix, A., Finlay, J. E., Abowd, G. D., y Beale, R. (2004). Human-computer interaction. Harlow: Pearson Education Limited.

Erbas, A. K., y Okur, S. (2012). Researching students' strategies, episodes and metacognitions in mathematical problem solving. Quality \& Quantity, 46(1), 89-102. doi:10.1007/s11135-010-9329-5

Ericsson, K. A., y Simon, H. A. (1980). Verbal report as data. Psychological Review, 87(1), 215-251.

Ericsson, K. A., y Simon, H. A. (1993). Protocol analysis. Verbal reports as data. Cambridge: MIT Press.

Forbes, I. (2001). The circle of understanding. Optimisation with the TI-92. Micromath, 17(3), 15-18.

García, J. N. (1993). Aplicación del análisis secuencial en la observación del desarrollo de los niños autistas. En Asociación Española de Profesionales del Autismo (Ed.), Actas del VII congreso nacional de autismo, "el autismo 50 años después de kanner” (pp. 163181). Salamanca, España: Amarú.

Gimeno, A., Anguera, M. T., Berzosa, A., Ramírez, L. (2006). Detección de patrones interactivos en la comunicación de familias con hijos adolescentes. Psicothema, 18(4), 785790.

Gluskin, R. (2004). Subtitle workshop (Versión 2.51) [Software de ordenador]. URUSoft. Obtenido de http://www.urusoft.net/products.php?cat=sw 
González, M. T., y Sierra, M. (2004). Metodología de análisis de libros de texto de matemáticas. Los puntos críticos en la enseñanza secundaria en España durante el siglo XX. Enseñanza de las Ciencias, 22(3), 389-408.

Goos, M., Galbraith, P., y Renshaw, P. (2002). Socially mediated metacognition: Creating collaborative zones of proximal development in small group problem solving. Educational Studies in Mathematics, 49(2), 193-223. doi:10.1023/A:1016209010120

Gorospe, G., y Anguera, M. T. (2000). Modificación de la técnica clásica de coordenadas polares mediante un desarrollo distinto de la retrospectividad: Aplicación al tenis. Psicothema, 2(2), 279-282.

Gorospe, G., Hernández, A., Anguera, M. T., y Martínez, R. (2005). Desarrollo y optimización de una herramienta observacional en el tenis de individuales. Psicothema, 17(1), 123-127.

Hernández, A. (1996). Observación y análisis de patrones de juego en deportes sociomotores. Santiago de Compostela: Servicio de publicaciones de la Universidad de Santiago de Compostela.

Jiménez, J. D. y Perales, F. J. (2000). Aplicación del análisis secuencial al estudio de texto escrito e ilustraciones de los libros de física y química en la ESO. Enseñanza de las Ciencias, 19(1), 3-19.

Kilpatrick, J. (1992). A history of research in mathematics education. En D. Grouws (ed.), Handbook of research on mathematics teaching and learning (pp. 3-38). New York: MacMillan.

Kapur, M. (2011). Temporality matters: Advancing a method for analyzing problem-solving processes in a computer-supported collaborative environment. International Journal of Computer-Supported Collaborative Learning, 6(1), 39-56. doi: 10.1007/s11412-0119109-9

Kursat, A., y Okur, S. (2012). Researching students' strategies, episodes, and metacognitions in mathematical problem solving. Quality \& Quantity, 46(1), 89-102, doi: $10.1007 / \mathrm{s} 11135-010-9329-5$

Lee, A. (2010). VirtualDub (Versión 1.9.11) [Software de ordenador]. Obtenido de http://www.virtualdub.org/

Lee, H. S. y Hollebrands, K. F. (2006). Students' use of technological features while solving a mathematics problem. The Journal of Mathematical Behavior, 25(3), 252-266. doi: 10.1016/j.jmathb.2006.09.005 
Lester, F. K. (1985). Methodological considerations in research on mathematical problem solving instruction. En E. Silver (ed.), Teaching and learning mathematical problem solving: Multiple research perspectives (pp. 41-69). Hillsdale, NJ: Lawrence Erlbaum.

Liao, C., Chen, Z. H., Cheng, H., \& Chan, T. W. (2012). Unfolding learning behaviors: a sequential analysis approach in a game-based learning environment. Research and Practice in Technology Enhanced Learning, 7(1), 25-44.

Malaspina, U. (2007). Intuición, rigor y resolución de problemas de optimización. Revista Latinoamericana de Investigación en Educación Matemática, 10(3), 365-399.

Malaspina, U. (2011). Resolución de problemas y estímulo del pensamiento optimizador en la educación básica. En A. Ruiz (Ed.) XIII Conferencia Interamericana de Educación Matemática (pp. II). Recife, Brasil: Comisión Interamericana de Educação Matemática. http://www.cimm.ucr.ac.cr/ocs/index.php/xiii_ciaem/xiii_ciaem/paper/viewFile/2818/ 1142

Ministerio de Educación y Ciencia [MEC] (2006). Real Decreto 1513/2006, de 7 de diciembre, por el que se establecen las enseñanzas mínimas de la Educación primaria. Boletín Oficial del Estado, n. 293, 43053-43102.

Ministerio de Educación y Ciencia [MEC] (2007a). Real Decreto 1631/2006, de 29 de diciembre, por el que se establecen las enseñanzas mínimas de la Educación Secundaria Obligatoria. Boletín Oficial del Estado, n. 5, 677-773.

Ministerio de Educación y Ciencia [MEC] (2007b). Real Decreto 1467/2007, de 2 de noviembre, por el que se establecen la estructura del bachillerato y se fijan sus enseñanzas mínimas. Boletín Oficial del Estado, n. 266, 45381-45477.

Mgombelo, J., y Bateau, C. (2010). Prospective secundary teachers repositioning by designing, implementing and testing mathematics learning objects: A conceptual framework. International Journal of Mathematical Education in Science and Technology, 8(40), 1051-1068. doi: 10.1080/00207390903236459

Muhr, T. (2010). In Scientific Software Development GmbH (Ed.), Atlas-Ti 6.0. Berlín:

National Council of Teacher of Mathematics [NCTM] (2000). Principles and Standar for school mathematics. Reston: NCTM.

Organisation for Economic Co-operation and Development [OECD] (2010). PISA 2012. Field Trial Problem Solving Framework. Obtenido de http://www.oecd.org/pisa/pisaproducts/46962005.pdf 
Organisation for Economic Co-operation and Development [OECD] (2014). PISA 2015. Draft Collaborative Problem Solving Framework. Obtenido de http://www.oecd.org/pisa/pisaproducts/pisa2015draftframeworks.htm

Perea, A. (2008). Análisis de acciones colectivas en el fútbol de rendimiento (Tesis Doctoral no publicada). Vitoria-Gasteiz: Universidad del País Vasco.

Poincaré, H. (1908). Science at méthode. París: Flammarion. Obtenido de http://www.acnancy-metz.fr/enseign/philo/textesph/Scienceetmethode.pdf

Pólya, G. (1945). How to solve it [Zugazagoitia, J. (1978), trad. Cómo plantear y resolver problemas. México, D.F.: Trillas]. Princenton: Princenton University Press.

Riba, C. (1993). Proceso de categorización. En M. T. Anguera (Ed.), Metodología observacional en la investigación psicológica. vol.1 fundamentación (pp. 115-168). Barcelona: Promociones y publicaciones universitarias.

Rico, L. (1997). Consideraciones sobre el currículo de matemáticas para educación secundaria. En L. Rico (Coord.), La Educación Matemática en la enseñanza secundaria (pp. 15-59). Barcelona: Horsori.

Sackett, G. P. (1980). Lag sequential analysis as a data reduction technique in social interaction research. En D. B. Sawin, R. C. Hawkins, L. O. Walker y J. H. Penticuff (Eds.), Exceptional infant. Psychosocial risks in infant-enviroment transactions (pp. 300340). New York: Brunner/Mazel.

Scher, D. (1999). Problem solving and proof in the age of dynamic geometry. Micromath, 15(1), 24-29.

Schoenfeld, A. (1985). Mathematical problem solving. Orlando: Academic Press.

Sharpe, T., y Koperwas, J. (2003). Behavior and sequential analyses. London: Sage Publications.

Symons, F. J., Hoch, J., Dahl, N. A., y McComas, J. J. (2003). Sequential and matching analyses of self-injurious bechavior: A case of overmatching in the natural environment. Journal of Applied Behavior Analysis, 36(2), 267-270. doi: 10.1901/jaba.2003.36-267

Törner, G., Reiss, K. M., y Schoenfeld, A. (2007). Problem solving around the world: summing up the state of the art. ZDM The International Journal on Mathematics Education, 39(5-6), 353-353. doi:10.1007/s11858-007-0053-0

Verderber, N. (1992). Tin cans revisted. The Mathematics Teacher, 85(5), 346-349.

Villegas, J. L., Castro, E., y Gutiérrez, J. (2009). Representaciones en resolución de problemas: un estudio de caso con problemas de optimización. Electronic Journal of Research in Educational Psychology, 7(17), 279-308. Obtenido de 
http://www.investigacion-

psicopedagogica.org/revista/articulos/17/espannol/Art_17_297.pdf

Yerushalmy, M. (2000). Problem solving strategies and mathematical resources: a longitudinal view on problem solving in a function based approach to algebra. Educational Studies in Mathematics, 43(2), 125-147, doi: 10.1023/A:1017566031373

Yimer, A., y Ellerton, N. F. (2006). Cognitive and metacognitive aspects of mathematical problem solving: An emerging model. En P. Grootenboer, R. Zevenbergen y M. Chinnappan (eds.), Identities, cultures, and learning spaces (pp. 575-582). Adelaida: Mathematics Education Research Group of Australasia.

Yimer, A., y Ellerton, N. F. (2010). A five-phase model for mathematical problem solving: Identifying synergies in pre-service teachers' metacognitive and cognitive actions. ZDM The International Journal on Mathematics Education, 2(42), 245-261, doi: $10.1007 / \mathrm{s} 11858-009-0223-3$

Yoder, P., y Symons, F. (2010). Observational measurement of behavior. New York: Springer. 